Aus der medicinischen Klinik zu Freiburg i. Br.

\title{
Ein unter dem Bilde eines Gehirntumors verlaufender Fall von chronischem idiopathischen Hydrocephalus in- ternus, complicirt mit symptomloser Syringomyelie,
}

\author{
mitgetheilt \\ von
}

\section{Dr. Heinz Kupferberg,}

ehemaligem Assistenzarzt der Klinik.

(Mit 10 Abbildungen.)

Schon der erste Theil der Uebersebrift der nachfolgenden $\mathrm{Pu}$ blication kiüdigt ein äusserst seltenes Krankheitsbild an; dessen im zweiten Theile erwähnte Complication bildet aber in dieser Form des Zusammenauftretens in der ganzen diesbezüglichen Literatur geradezu ein Unicum. Hieraus mag sich zu unserer Entschuldigung ergeben, weshalb wir es für nöthig hielten, selbst auf die Gefahr hin, den Leser zu ermüden, einestheils unseren Fall so ausführlich zu publiciren, und anderentheils auch die ihm etwa parallelen Fälle aus der Literatur ihm kurz im Auszuge zur Seite zu stellen. Auch was sonst in der Literatur von solehen, dem unseren ähnlichen Fällen gefunden werden konnte (namentlich in Bezug auf die Complication mit anderen Anomalien im Centralnervensystem), möge zum Vergleiche kurze Erwähnung finden.

Trudpert Lorenz, 48 Jahre alt, Steinbrecher, am 24. Februar 1891 in die medicinische Klinik in Freiburg i. Br. aufgenommen. nommen).

Anamnese (zum grössten Theil von der Fran des Patienten aufge-

I. Allgemeine anamnestische Angaben:

a) der Familie.

Die Eltern des Patienten sollen beide an "Lungenentzündung" gestorben sein. Fünf Geschwister des Patienten leben und sind gesund; ein 
Bruder vor sechs Jahren an „Wassersucht" gestorben. - Nerven- oder Geisteskrankheiten sind in der Familie des Pat. niemals vorgekommen.

b) des Patienten selbst.

Als Kind fiel Pat. von einem Heuboden in den Futtergang hinab, wobei er sich eine Verletzung der Unterlippe zuzog, die genäht werden musste (Spuren davon noch zu sehen); ob damals eine Gehirnerschütterung oder Schädelverletzung bestanden, lässt sich nicht eruiren; jedenfalls war er eine Woche nach dem Unfall wieder völlig gesund.

1864 hat Patient die Diphtheritis durchgemacht, die ihn acht Tage an das Bett fesselte; danach war er wieder völlig gesund.

1865 will Pat. längere Zeit an der "Ruhr" krank gelegen haben.

1866 soll er sich durch das Platzen einer Schlagröhre (er genügte seiner Dienstpflicht bei der Artillerie zu Rastatt) eine Angenverletzung zugezogen haben, die nach 14 tägiger Behandlung im Lazareth völlig und ohne Hinterlassung von Sehstörungen geheilt sei.

18\%0/71 machte Patient den Feldzug mit und bekam während desselben Brechdurchfall, weshalb er 12 Wochen lang im Hospital zu Rastatt lag; danach war er wieder völlig gesund, nur war und blieb er seit dem Feldzug schwerhörig auf dem rechten Ohr.

1877 verheirathete sich Patient; seine Frau, die gesund ist, hat $13 \mathrm{mal}$ geboren, niemals Fehl- oder Frühgeburten gehabt; von den 13 Kindern sind 12 gestorben (5 innerhalb eines Jahres an „Lnngenschlag“" im Alter von 1-6 Jahren, 1 an „Wasser im Hirn“, und die 6 anderen an „Brechdurchfall" in den ersten Lebensmonaten).

1885 lag Pat. an einer Augenverletzung längere Zeit in der hiesigen Augenklinik; eine zum Sprengen im Steinbruche benutzte Röhre entlud sich zu früh, und das zur Erhöhung der Sprengwirkung auf dieselbe gegossene Wasser spritzte $\mathrm{ihm}$ zugleich mit den Pulverkörnern in das Gesicht. Die Diagnose der Verletzung lantete damals, laut Krankengeschichte der hiesigen Augenklinik: Conjunctivitis traumat.; Sugillat. conj. palpeb. et bulbi; Keratit. traumat. bilat. - Nach viermonatlicher Behandlung dort (zuerst klinisch und dann ambulatorisch) wurde er fast völlig geheilt entlassen mit S. L. ${ }^{4} / 6$ o. D. ; S. R. $3 / 6$ o. D. entsprechend den noch leichten restirenden oberflächlichen Trübungen, besonders auf der rechten Cornea; im Augenhintergrund damals nichts Abnormes. Seit dieser Verletzung will Pat. nicht mehr so gut sehen können, und seine Fran giebt an, man habe ihm dies auch an seinen Augen angemerkt, „die seitdem immer etwas trübe und matt ausgesehen hätten".

1890 (im Januar) will Patient zweimal leichte Influenzaanfälle durchgemacht haben; er lag dabei nicht zu Bett, und acht Tage später will er wieder ganz gesund gewesen sein, - seine Fran weiss jedoch nichts davon, dass der Patient je die Influenza gehabt.

1890 (im Herbst) wurde ihm beim Steinladen die Endphalanx des 4. Fingers der rechten Hand abgequetscht, und befand er sich deshalb (Reamputation) in der hiesigen ehirurgischen Poliklinik acht Tage lang in ambulatorischer Behandlung, wonach er geheilt entlassen wurde.

1890 (im Spätherbst) bekam Patient das "Gliederweh mit Fieber" (herumwandernde Schmerzen in den Arm- und Beingelenken und im Rücken, 
mit Unvermögen, sich zu bücken); er habe weisse Pulver zum Schwitzen and etwas zum Einreiben bekommen und sei nach dreiwöchentlichem Krankenlager wieder ganz gesund gewesen; in der Reconvalescenzperiode wurde er auch zweimal am Rücken elektrisirt. Nach Aussage des damals behandelnden Arztes handelte es sich bei dem Pat. um einen acuten Gelenkrheumatism us ohne Herzerkrankung.

\section{Anamnese zu der jetzigen Krankheit.}

Schon seit seiner Verheirathung (1877) habe Patient öfter über Schmerzen im Rücken (Kreuz) geklagt, und wenn er sich bei der Arbeit gebückt, sei es ihm hie und da schwindelig und schwarz vor den Augen geworden; doch war er stets ein kräftiger Mann und fleissiger Arbeiter. Seit fast einem Jahre hatte Patient oft über allgemeine Mattigkeit zu klagen.

Vor acht Jahren (1883) konnte Patient eines Nachts plötzlich das Wasser nicht lassen, und der zugezogene Arzt musste damals und noch 14 Tage nachher täglich einmal katheterisiren, was dann die Frau des Pat. noch weitere 14 Tage lang fortsetzen musste; er lag damals im Ganzen acht Wochen zu Bett. Seitdem sei stets eine gewisse Erschwerung beim Wasserlassen vorhanden gewesen, und hie und da sei auch einmal gegen den Willen des Pat. Urin abgeflossen.

Seit dem oben erwähnten Gelenkrheumatismus (1890) ging Pat. nicht mehr in den Steinbruch (nicht etwa weil er sich krank fühlte, sondern weil im Winter dort die Arbeit ruht), nahm aber eine Stelle als Aufseher an einem Eisweiher an, wo er auch Nachtwachen that; er fühlte sich während dieser Thätigkeit stets ganz wohl.

An einem Vormittag Anfang Januar 1891 glitt Pat. bei seinem Dienste auf dem Eise aus und fiel auf den Hinterkopf. - Ob er danach bewusstlos wurde, weiss die Frau nicht mehr anzugeben; er kam jedoch wie gewöhnlich zum Essen nach Hause, klagte nur über leichtes Kopfweh und ging nach Tisch wieder auf den Eisweiher; erst als er Abends (Feierabend machend) nach Hause $\mathrm{kam}$, sagte er seiner Frau, er habe ein Gefühl, als ob ihm „der Hinterkopf abgeschlagen sei". Am nächsten Tage aber that er seinen Dienst weiter und war geistig und auch sonst unverändert; nur das Kopfweh bestand fort.

Am 20. Januar 1891 Vormittags $11 \mathrm{Uhr}$ fühlte er plötzlich, als er aus seiner Wohnung in den Keller gehen wollte, Schwindel und musste an der Wand tastend und sich stützend sein Bett aufsuchen, wo er drei Stunden sich ausruhte (er war etwas schläfrig); um 2 Uhr Nachmittags kommt er in die Küche heraus zu seiner Frau, um sie etwas zu fragen, als er plötzlich, ehe er fragen konnte, rü ckwärts umstürzte und mit dem Hinterkopf anf die Ecke des Herdes schlug, so dass er blutend aus einer kleinen Wunde hinter dem rechten Ohr auf dem Rücken lang gestreckt regungs- und bewusstlos liegen blieb. Er wurde in das Bett getragen und kam dort nach einigen Minnten wieder zu sich; er klagte über heftige Schmerzen im Nacken und Hinterkopf, den er beständjg mit beiden Händen hielt; sonst war er ganz klar, sprach und bewegte sich wie immer und hatte kein Erbrechen, keinen Schwindel und keine Krämpfe. Abends kam der Arzt, der ein Senfpflaster auf den 
Nacken legte und für die folgenden Abende je ein Fassbad verordnete. Der Zustand des Pat. blieb drei Tage lang unverändert. In der dritten Nacht (vom 22. auf 23. Januar) aber bekam er "Krämpfe", eingeleitet durch Erbrechen, Umsinken, Bewusstlosigkeit, völige Streckstarre der Arme und Beine, Opisthotonus und Genickkrampf; die Augen waren nach oben gerichtet, so dass nur das "Weisse" zu sehen war; mit den Füssen geschahen zeitweise zuckende und schlagende Bewegungen. Dieser Anfall dauerte fünf Minuten. Ein sofort herbeigeholter Arzt setzte elf Blutegel und ein Senfpflaster in den Nacken. Kaum war der Arzt wieder fort, als der gleiche Krampf sich wiederholte, eingeleitet von Erbrechen und genau wie oben verlaufend. Noch 3 mal kam dann der gleiche Krampfanfall in der Nacht. In den freien Pausen füblte sich Pat., ausser heftigen Schmerzen im Hinterkopf und Schwindel, ganz wohl, so dass er am nächsten Morgen den Arzt in dessen Wohnung aufsuchen konnte. - Vom 23. Januar bis 24. Februar hatte Pat. an jedem Abend zwisehen 6 und $8 \mathrm{Uhr}$ „die Krämpfe" mit völliger Bewusstlosigkeit, eingeleitet durch Erbrechen; das Erbrechen trat jedoch auch tagsüber a uf (vom 23. Januar ab), und zwar 3- bis 4 mal täglich, oft 2 mal innerhalb einer Stunde. In den freien Pausen war Pat. aber durchaus normal, nur klagte er viel über Kopfweh in der Stirn und im Hinterhaupt; Schwindel, resp. schwankender Gang sei an ihm nicht bemerkt worden. Am 27. Januar 1891 vermuthete der behandelnde Arzt bereits eine wachsende Geschwulst im Gehirn (eine Augenspiegeluntersuchung war jedoch noch nicht vorgenommen worden), und verordnete verschiedene Arten von Pulvern und Mixturen; doch bestanden Kopfschmerzen und Erbrechen in gleicher Weise fort, ebenso wie die allabendlich eintretenden Krämpfe. Erst am 24. Februar suchte Pat. die hiesige Klinik auf, obschon dies der Arzt bereits vier Wochen vorher angerathen, wogegen aber der Pat. stets sich sträubte, vorgebend, , lieber zu Hause sterben zu wollen". - Er ging vom 2. Stock seiner Wohnung die Treppe hinunter bis za der vor seiner Hausthür wartenden Droschke und in der Klinik von der Droschke wieder bis in den 3. Stock hinauf, ganz ohne Hülfe und ohne Stock.

\section{Allgemeine anamnestische Schlussbemerkungen.}

Lues oder Gonorrhoe will Pat. niemals durchgemacht haben. Früher habe Pat. viel getrunken, und sei er oft betrunken nach Hanse gekommen, wobei er dann sehr roh und verwirrt gewesen sei. Nüchtern soll er jedoch „der beste Mensch" gewesen sein; klng und fleissig habe er stets die Zufriedenheit seiner Arbeitgeber erworben. Besondere manuelle Geschicklichkeit habe er nicht besessen. Im Rechnen, Lesen und Schreiben sei er nie hervorragend gewesen, doch von grosser Gewissenhaftigkeit und mit gutem Gedächtniss begabt bis zur jetzigen Erkrankung, wo letzteres sehr abgenommen habe. Pat. war stets leicht erregbar, sehr jähzornig, und dann oft nicht mehr Herr seiner Handlungen. Ueber sein Seh- und Hörvermögen vgl. oben; Geschmack-, Geruch- und Gefühlssinn seien stets normal gewesen. Schwindel, Parästhesien (Kribbeln im Rücken oder in den Beinen), Zittern, Kopfschmerzen, Erbrechen, 
oder irgend welche Motilitätsstörnngen habe er vor der jetzigen Erkrankung (20. Januar 1891) niemals gehabt. - Der Stuhlgang sei früher stets in Ordnung gewesen; und auch jetzt sei nur öfter etwas Verstopfung vorhanden; das Wasserlassen (vgl. oben) sei durch die jetzige Krankheit nicht beeinflusst. - Seit Ende Januar 1891 habe er stets etwas Husten und schleimigen, gelblich gefärbten Auswurf. - Pat. lebte in ganz guten Verhältnissen, mit einfacher, aber kräftiger, bürgerlieher Kost. Starken körperlichen Anstrengungen, sowie Erkältungen und Durchnässungen war er in der Ausübung seines Berufes des Oefteren ansgesetat.

Ausser dem früher erwähnten Gelenkrheumatismus will Pat. Gelenkerkrankungen niemals durchgemacht haben.

Status praesens. Inspection. 24. Februar 1891. Patient nimmt die active Rückenlage ein; bisweilen leises Stöhnen, schmerzhaftes Verziehen des Gesichts und Fassen der Hand nach der Stirn.

Musculatur kräftig entwickelt, ohne Abnormitäten oder Differenzen zwischen links und rechts.

Fettpolster mässig stark, Knochenbau sehr stark, Hals etwas kurz, Statur gedrungen; Kopf ziemlich gross, etwas viereckig. Mittlere Körpergrösse; Körpergewicht $70 \mathrm{Kgrm}$.

Nirgends Exantheme oder deren Reste, Oedeme, Gelenkerkrankungen, varicöse Erweiterungen, Drüsenschwellungen oder Narben; an den Knochen keine Auftreibungen. Schleimbaut des Mundes und Rachens ohne Veränderungen. Gesichts- und Hautfarbe gesund, keine Anämie; ganz leichter Icterus der Sclerae; Gebiss sehr defect; einzelne Zähne cariös (mehrere fehlen), einzelne hauerartig missbildelt.

Sensorium frei; Psyche: leichte Depression, etwas Apathie; allen Aufforderungen wird langsam Folge geleistet, alle Fragen etwas langsam, aber durchaus logisch richtig beantwortet; es tritt auf alle Fragen und Aufforderungen erst eine Pause des Nachdenkens ein, ehe darauf reagirt wird. Gedächtniss etwas beeinträchtigt, jedoch keine gröberen Störungen. Leichte Rechenaufgaben werden ganz gut aus dem Kopfe gelöst.

Sprache etwas langsam, sonst völlig normal; Stimme klar, nur etwas leise.

Innere Untersuchung.

Körpertemperatur: $36,5^{0}$ (in der Achselhöhle).

Puls : 60-64, voll, kräftig, regelmässig.

Respiration: 14-16, tief, regelmässig, von normalem Typus.

Lungen: Untere Grenzen etwas tiefer als normal, bei der Respiration gut verschieblich, nirgends Dämpfung, überall etwas verschärftes Inspirium; verlängertes Exspirium. H. U. beiderseits spärlich feuchtes Rasseln, vereinzelte Rhonchi; Sputum: mässig reiehlich, katarrhaliseh, ohne Tuberkelbacillen.

Herz: Dämpfung beginnt an der 4. Rippe, geht nach rechts bis zum linken Sternalrand, nach links bis fast zur Papillarlinie. Spitzenstoss an normaler Stelle, schwach fühlbar. Herztöne rein. Zweiter Pulmonalton leicht verstärkt. An den Halsvenen leichte präsystolische Undulation und exspiratorische Staung. - Puls s. oben.

Abdomen leicht meteoristisch aufgetrieben, ohne Fluidum und ab- 
norme Resistenzen. - Leber in der rechten Papillarlinie von dem VII. Intercostalranm bis zum Rippenbogen reichend nicht palpabel.

Milz. Dämpfung nicht vergrössert. Organ nicht palpabel.

Kopf. Beklopfen des Kopfes, besonders in der Scheitelgegend, sehr schmerzhaft, Compression oder Beklopfung der Wirbelsäule nirgends schmerzhaft; dagegen die Belgung des Kopfes nach vorn (siehe später).

$$
\text { Muskeln, Motilität. }
$$

M uskeln überall kräftig entwickelt, von normalem Spannungszustand (keine Spasmen oder Contracturen); nirgends Tremor; keine fibrillären Zuckungen. Bewegungen der Bulbi und Lider normal; im Facialisgebiet überall normale Motilität, keine Differenzen zwischen links und rechts, nur erscheint die Unterlippe links etwas vorgewulstet, was jedoch auf ein in der Kindheit erlittenes Trauma (s. Anamnese) zurückzuführen ist. $\mathrm{Zunge}$ wird gerade herausgesteckt, beiderseits gleich dick (ohne fibrilläre Zuckungen) und frei nach allen Seiten beweglich. Gaumen und L a ry $n$ muskeln zeigen normale Motilität; Schling a et normal. Beugung des Kopfes nach vorn sowohl activ wie passiv nur in geringem Maasse möglich (etwas Nackenstarre); bei dem Versuche passiver Beagung heftige Schmerzen im Nacken und Hinterkopf. - An den Armen passive Bewegungen in allen Gelenken völlig frei, active völlig normal und kräftig, Händedruck beiderseits gleich kräftig. $\mathrm{R}$ u $\mathrm{p}$ fmuskeln normal beweglich.

An den Beinen erscheint die active Beweglichkeit etwas beeinträchtigt; es macht sich eine gewisse Unsicherheit und Schwäche dabei bemerkbar; passive Bewegungen in allen Gelenken frei.

\section{Reflexe.}

Sehnenreflexe an den Patellarsehnen vielleicht etwas gesteigert, beiderseits gleich (kein Kniephänomen); an den Achillessehnen dentlicher Reflex (kein Fussphänomen); tiefe Reflexe (Periost) undeutlich.

Hautreflexe überall völlig normal (Znckungen prompt), auch Bauchdecken- und Cremasterreflex.

Muskelsinn und Muskelgefühl erscheinen in den Beinen vielleicht etwas herabgesetzt (jedoch keine deutlichen Ataxien oder Störungen des Orientirtseins über die Lage und Haltung seiner Extremitäten), sonst durchaus normal.

Mechanische Erregbarkeit der Muskeln überall normal, prompt.

Die elektrische Untersuchung ergiebt sowohl für den faradischen, wie für den galvanischen Strom vom Muskel und Nerven aus tuberall durchaus normale Resultate.

Blase und Mastdarm functioniren ziemlich normal, Wasserlassen nur öfter etwas erschwert, Stuhl leicht angehalten (vgl. hierzu Anamnese).

\section{Sensibilität.}

Die Sensibilitä der Haut ist in allen ihren Qualitaten und an allen Körperstellen völlig normal, auch bestehen keine Parästhesien, keine Verlangsamung der Leitung sensibler Reize. Eine ein- 
gehendere Prüfung ist dadurch erschwert, dass es nicht leicht gelingt, die Aufmerksamkeit des Pat. längere Zeit auf diese Prüfungen zu fixiren. Betreffs der Sensibilität der Muskeln siehe unter Muskelgefühl.

Der Gang des Pat. ist etwas unsicher und schwankend wegen starken Schwindel gefühls, wodurch sich wohl (zum grössten Theil wenigstens) auch das dentlich ausgesprochene Brach-Rombergsche Pbänomen erklärt, zeigt aber sonst keine Besonderheiten. - Auch beim Aufsitzen hat Pat. Sehwindelgefühle und etwas Schwanken im Oberkörper bei geschlossenen Augen.

\section{Sinnesorgane.}

A ugen: Bewegungen der Bulbi und Lider durchans normal, coordinirt; kein Nystagmus; Pupillen mittelweit, beiderseits gleich, reagiren etwas träge a uf Lichteinfall, auf Accommodation und Convergenz normal.

Sehschärfe auf beiden Angen sehr stark herabgesetzt; nicht corrigirbar. (Pat. giebt an, er sähe Alles wie durch einen Schleier oder ,als habe ex einen Nebel vor den Augen"; vgl. Anamnese.) Gesichtsfeld nicht genauer zir prüfen, zum Theil anch wegen der Apathie und mangelhaften Aufmerksamkeit des Patienten. Farbensinn nur entsprechend der mangelhaften Sehschärfe beeinträchtigt.

Ophthalmoskopie: Beiderseits exquisite Staungspapille (Papille vorgewölbt, granstreifig verfärbt, Ränder verwaschen, Venen starkerweitert) und zahlreiche kleinere und grössere ziemlich frische Hämorrhagien in der Peripherie beiderseits; links am die Papille eine grössere hufeisenförmige Hämorrhagie, rechts an der Macula mehrere grössere unregelmässig gestaltete Hämorrhagien.

O hren: Hörvermögen rechts etwas herabgesetzt (vgl. Anamnese), links normal, Leitung dureh die Kopfknochen beiderseits normal.

Otosk opi e: rechts gleichmässige diffuse Trübung des Trommelfells, links nichts Abnormes.

Geruchsinn des Pat. scheinbar normal.

Geschmack nicht, oder nicht wesentlich beeinträchtigt.

Stuhl geformt, ohne pathologische Beimengungen (vgl. Anamnese).

Harn wird in normaler Tagesmenge entleert, reagirt schwach sauer, ist klar und frei von abnormen chemischen und geformten Bestandtheilen; spec. Gewicht 1012-1014.

Klinischer Verlauf: 25. Februar. Pat. hat im Laufe der Nacht 3 mal erbrochen, ebenso heute morgen (im Erbrochenen nichts Abnormes; zwei Stunden nach der Mahlzeit: Erbrochenes riecht und reagirt stark sauer, enthält aber keine freie Salzsäure). - Kopfschmerzen etwas geringer. - Verordnung: Magenausspülung. $2 \square$ Blasenpllaster in den Nacken.

27. Februar. Stuhlverstopfung; da anf Calomel $(2 \times 0,3)$ kein Stuhl erfolgt, Abends Einlauf. Stuhl geformt, ohne pathologische Beimengungen. - Heftige Kopfschmerzen, deshalb Abends 8 Milligrm. Morphin subcutan. Gestern und heute 2 mal erbrochen. 
2. März. „Krämpfe" bisher nicht eingetreten. Kopfschmerzen unverändert; 1,0 Antipyrin p. Clysma dagegen ohne Erfolg. Fast täglich 1-2mal Erbrechen. - Stublverstopfung; 30,0 inf. semn. comp.; Nachts 12 Uhr "Anfall": Beginn ohne Aura; Bewusstlosigkeit, klonische Beugekrämpfe in beiden Armen (wobei schmerzhaftes Stöhnen eintritt), 3-4 Minuten danernd. Buibi beide $n$ a ch oben a bgelenkt, Pupillen reactionslos, mittelweit; Lid- und Patellarreflexe erhalten, Hautreflexe nicht hervorzurufen.

3. März. Heute $3 \mathrm{mal}$ die gleichen "Anfälle", ohne Aura; nachher heftige Kopfschmerzen; 6 Mgrm. Morph. sube.

6. März. Seither kein "Anfall". Erbrecben dauert fort, täglich 2-3 mal durchschnittlich, unabhängig von der Nahrungsaufnahme. Ord.: Kal. jodat. $3 \mathrm{mal}$ täglieh 0,4 .

8. März. Erbrechen etwas nachlassend; subjectives Befinden besser; weniger Schwindelgefühl, fast keine Kopfschmerzen.

10. März. Bronchitis im Zunehmen; sonst stat. idem. Kal. jodat. und Liq. Ammon. anisat. $\overline{\text { à }}$ 5,0. Aqu. dest. 180,0. M. D. S. 3 mal tägl. 15,0.

11. März. Bisherige Gewichtsabnahme: 6 Pfd. Patient giebt an, öfter am Tage vorübergehend die Empfindnng zu haben, als ob ihm plötziich ein schwarzes Tuch über die Augen gezogen würde (vorübergehende Obscurationen); auch merke er eine stetige Abnahme seines Sehvermögens. - Ophthalmoskopie ergiebt den früheren Befund.

12. März. Heftige Kopfschmerzen; linke Pupille bedentend weiter als rechte; Reaction beiderseits träge auf Lichteinfall; links leichte Ptosis, doch vermag das linke Oberlid noch ganz gehoben zu werden. - Nach einer halben Stmnde Nachlassen der Kopfschmerzen, beide Pnpillen wieder gleich weit.

14. März. Heftige K opf- und Nackenschmerzen; Erbrechen besteht fort; öfter Obscurationen auf Augenblicke.

16. März. Nackensebmerzen vorüber, sehr h eftig e K op f se h merzen, plötzlich einsetzend; linke Pupille dabei sehr weitwerdend, reactionslos; Mnsc. rect. ext. des linken Auges insufficient.

17. März. Morgens beide Pupillen gleich weit. Nachmittags heftige K opfschmerzen, plötzlich einsetzend; während derselben linke $\mathrm{Pu}$ pille weiter als rechte und träger auf Lichteinfall reagirend. Patient giebt an, hente viel sehlechter zu sehen; auf 1 M. Entfernung vermag Patient kaum noch Finger zu zählen (beiderseits).

18. März. Heftige Scbmerzen in Nacken und Kopf; linke Pupille weiter, reactionslos. Bronchitis im Zunehmen. Sol. kal. jodat. 5,0: 180,0 . M. D. S. 3 mal tägl. 20,0 .

20. März. Erbrechen besteht fort. Pupillen heute gleich weit und von gleich träger Reaction. Abends $1 / 2$ stïndiger Anfall von Bewusstlosigkeit ohne sonstige Störungen. Danach heftige Kopfschmerzen, linke Pupille weiter, reactionslos. Im Harn ausser etwas vermehrter Indican-Ausscheidung nichts Abnormes. Stuhl dauernd etwas angehalten.

23. März Abend̄s 5 Uhr. 1/4 stündiger Anfall von Bewusstlosigkeit; dabei Strabismus divergens: rechtes Auge nach unten und aussen, linkes Ange geradeaus gerichtet. Linke Pupille weiter 
als rechte, beide reactionslos. Links zeitweise klonischer Facialiskrampf, sonst nirgends Krämpfe. Extremitäten schlaff. Nach dem Anfall heftige Stirn- und Nackenschmerzen; linke Pupille weiter als rechte, reactionslos. Strabismus und Facialiskrampf verschwunden.

25. März. Ueber den unteren Lungenpartien beiderseits reichlich feuchtes Rasseln, keine Dämpfung; Sputum ziemlich reichlich, schleimigeitrig, leicht expectorirt. - Kal. jod. ausgesetzt.

1. April. Pupillen gleich weit, gleich träge reagirend. Kopfschmerzen geringer, Erbrechen besteht fort.

Abends: Pat. etwas benommen, klagt über sehr heftige Ko pfs ch mer zen; linke Pupille weiter, reactionslos. - Gesichtshälften symmetrisch beiderseits, Zunge wird gerade herausgestreckt, ohne Tremor. Nackenstarre geringer. Heftige Schmerzen bei passiven Bewegungen des Kopfes. Beklopfen des Scheitels äusserst schmerzhaft. Bulbusbewegungen (deren Prüfung wegen der nahezu völligen Amaurose sehr erschwert) nicht genau coordinirt. Beim Blick nach links bleibt das linke Auge etwas zurü ck und macht leichte horizontale nystagmusartige Zuckungen. - Patellarreflex rechts vielleicht etwas gesteigert, rechts auch Andeutung von Fussphänomen.

Einige Male läuft unfreiwillig Harn ab; andere Male wieder besteht Unfähigkeit Harn zu lassen, obwohl starker Harndrang und die Blase nachweisbar gefüllt ist.

2. April. Erbrechen besteht fort, täglich 1-2mal; selten ein Tag ohne Erbrechen. Mehrere Tage hindurch fortgesetzte Beobachtungen des Verhältnisses von Puls, Athmung, Pupillenerweiterung and Pupillenreaction zu der Intensität der Kopfschmerzen ergiebt: Bei keinen oder geringen Kopfschmerzen: Puls 76-80, Athmung 20-24, Pupillen gleich weit, gleich träge reagirend (vielleicht links etwas träger); bei heftigen Kopfschmerzen, die meist anfallsweise anftreten: Puls $60-70$, Athmung 20, Pupillen ungleich (linke weiter), reactionslos.

4. April Abends 6 Uhr. Pat. reagirt nicht anf Anrufen; lässt Harn unter sich; bei der Athmung ,Tabaksblasen"; Cheyne-S tokes. - Harn ohne Eiweiss. Nach $1 / 2$ Stunde Rückkehr des Bewusstseins, heftige Kopfschmerzen; Puls 60, linke Pupille weit, reactionslos. Athmung regelmässig.

6. April. Pat. sehr a a thisch; auf Fragen giebt er keine Antwort, sondern wiederholt nur langsam die Frage mit leiser tonloser Stimme.

7. April. Linksseitige Ptosis deutlicher; starke Kopfschmerzen. Insufficienz des Musc. rect. ext. des linken Auges deutlicher. Seit einigen Tagen völlige Incontinentia urinae.

11. April. Ptosis links zunehmend; im rechten Facialis (in allen Theilen) deutliche Parese ${ }^{1}$, linke Pupille weiter als rechte, beide reactionslos. Zunge wird etwas nach rechts

1) Leider ist die spätere elektrische Untersuchung des paretischen Facialis versäumt worden, daher wichtig, dass auch der Stirntheil des Facialis paretiseh war. 
herausgestreckt, erscheint links etwas abgeflacht. - Gedächtniss und Sprache unverändert. - Beginnender Intertrigo am Scrotum und zwischen den Oberschenkeln infolge der Incontinentia urinae. Am Gaumen keine Paresen. Täglich Sitzbad. Salicyl-Talkumeinpuderung.

Abends 7 Uhr: ,Anfall". Bewusstlosigkeit. Extremitäten schlaff, Cornealreflex vorhanden. Klonischer Krampf im Musc. rect. ext. des rechten Auges.

13. April. Nackenstarre wesentlich zurückgegangen. Nachmittags 4 Uhr: leichter $1 / 4$ stündiger Anfall von Bewusstlosigkeit. Danach heftige Kopfschmerzen.

Abenas 6 Uhr: "Anfall." Kopf stark tonisch in den Nacken gebeugt, völlige Bewusstlosigkeit, stertoröses Athmen. - Auf äussere Reize kehrt nach 5 Minuten das Bewusstsein wieder. Pat. giebt an, nicht mehr gut zu schmecken: Chinin wird erst nach dem Schlucken als bitter erkannt, Salz als sauer bezeichnet, Zncker gar nicht erkannt.

Erbrechen besteht nach wie vor.

Links Ptosis deutlicher, linke Pupilleweiter als rechte, beide reactionslos.

16. April Abends. Linker Mundwinkel etwas nach links gezogen, rechts Gesichtsfalten deutlicher verstrichen.

Pat. etwas unklar; giebt an gehen zu können; bei einem Versuche tritt sehr starker Schwindel ein und zeigt sich völlige Unfähigkeit zu gehen, wenn Pat. nicht von beiden Seiten festgehalten wird. Sensibilität nirgends, wenigstens gröber gestört. Patellarreflexe beiderseits etwas verstärkt, kein Fussclonus. - B e i d e P u pillen gleich weit, reactionslos.

17. April Morgens: Linke Papille bedeutend weiter als rechte, beide ohne Reaction.

Abends: Leichter Strabismus divergens des rechten Auges. Pat. etwas unklar. Ptosis links zunehmend, ebenso Facialis Parese rechts. Linkes Auge vermag nicht über die Mittellinie nach rechts gedreht zu werden.

Patient lässt 2 mal Stuhl unter sich bei einigermassen erhaltenem Bewusstsein. Incontinentia urinae besteht fort. - Intertrigo zunehmend. Täglich warmes Vollbad. Salicyltalkeinpuderung.

21. April Abends. 1/4stündiger Anfall von Bewusstlosigkeit. Pat. lässt einmal Stuhl unter sich. Harn ohne Eiweiss.

23. April Abends. Rechter Bulbus etwas nach rechts und oben abgelenkt, linker geradeaus gerichtet; linke Pupille weiter als rechte, reactionslos; Athmung tief, regelmässig. Puls $60-70$, regelmässig. blasen".

27. April. Athmung regelmässig, beim Exspirium etwas ,Tabaks-

Fast täglich einmal seit dem 21. April kurzer Anfall von Bewusstlosigkeit; Pupillen dabei weit, reactionslos. Pat. lässt stets Stuhl unter sich. Erbrechen besteht fort. - Harn ohne Eiweiss und Zucker.

1. Mai. Klinische Vorstellung: Pat. klagt über dumpfen Kopfs chmerz, dessen Sitz hauptsächlich die Stirngegend sei. Sprache zeigt weder ataktische, noch aphasische Störungen, Antw ort en a f alle Fra- 
gen prompt und richtig, Stimme etwas leise, belegt. Es besteht ein geringer Grad von Nackenstarre. Beim Aufsitzen stärkeres Schwindelgefuhl, doch etwas geringer als früher.

Beide Arme werden normal bewegt und gehoben, mit den Händen ein ganz guter Druck ausgeübt; keine Differenz zwischen links und rechts. Ernährungszustand der Muskeln, der oberen und unteren Extremitäten gut, keine Differenzen zwischen links und rechts. Die Beinewerden im Bette ebenfalls ganz gut bewegt; keine ansgesprochene Parese, keine Differenz zwischen links und rechts. Gang wegen heftigen Schwindels unmöglich.

Die Falten der rechten Gesichtshälfte erscheinen weniger ausgeprägt, besonders die Nasolabialfalte; links besteht leichte Ptosis, doch kann das linke obere Augenlid noch gehoben werden. Patient ist schon seit einigen Wochen (Mitte April) vöilig a maurotisch, daher die Bulbusbewegungen nicht genauer za untersuchen. Es besteht ein leichter Grad von Strabismus divergens des linken Auges; die linke Pupille deutlich weiter als die rechte. Bei längeren Beobachtungen zeigt sich ein beständiger Wechsel in der Weite der Pupillen, doch besteht völlige reflectorische Pupillenstarre. Die Bnlbi werden beständig etwas unstät hin und her bewegt. Beim Blick nach rechts wird der rechteBulbus nicht ganz nach aussen bewegt, dagegen kann beim Blick nach links der linke Bulbus ganz nach links bewegt werden. Nach links folgt das rechte Auge gut, ebenso nach rechts das linke Auge bis zur Mittellinie. Beim Blick nach oben bleibt das linke Auge deutlich zurück. Beim Blick nach unten kein Unterschied. Beim Blick nach rechts bleibt zeitweise der linke Bulbus etwas zuruek, und es tritt leichter Nystagmus dabei anfo

Zunge wird gerade herausgestreckt, kann frei nach beiden Seiten bewegt werden, erscheint jedoch links etwas flacher; die Vertiefungen an dem Zungenrand entsprechen restirenden, hanerartig vorspringenden Zähnen (Gebiss sehr defeet).

Beim Pfeifen und Blasen tritt deutlich ein etwas mangelhafter Schluss der rechten Mudhälfte hervor.

Die ophthalmoskopische Untersuchung ergiebt:

Linke Pupille exquisit vorgew ölbt, streifig getrübt, umgeben von einer grossen, hufeisenförmigen älteren Hämorrhagie; in der Umgebung bis zur Peripherie zahlreiche ältere, kleinere und grössere Hämorrhagien. - Rechte Papille vollständig verschwommen, kaumzuerkennen; an der Maculagrosseältere Hämorrhagien; Umgebung bis zur Peripherie wie am anderen Auge. Die Diagnose wird auf einen Tumor im Mittelhirn gestellt. Vgl. weiter unten.

2. Mai. Beim Blick nach rechts bleibt der rechte Bulbus etwas zurück, der linke stärker (Strabismus divergens des Iinken Auges); dabei tritt links Nystagmas ein. Beim Blick nach links heute ebenfalls links Nystagmus. Ueberhaupt erscheint heute die Bewegung des linken Bulbus mehr eingeschränkt, als 
gestern; a ch die Ptosis und grössere Pupillenweite links sehr deutlich.

Sehnenreflexe: Patellarreflex beiderseits gleich, nicht gesteigert; Muskeln völlig schlaff, kein erhöhter Tonus, Fussphänomen weder rechts noch links vorhanden. Achillessehnenreflex beiderseits normal.

4. Mai Morgens: Klagen über heftigere Kopfsehmerzen; seit zwei Tagen viel Schlafsucht; leichte Benommenheit.

Abends: Pat. mehr benommen; schwitztstark, aber beiderseits gleich. - Bronchitis zunehmend. - Oculomotorius-Parese links deutlicher.

5. Mai. Pat. schluckt nicht mehrgut; Benommenheitzunehmend. Aussehen etwas verfallen.

6. Mai Abends: B enommenheit nimmtzu; auf Anrufen und äussere Reize reagirt Pat. nur durch müden Augenaufschlag.

7. Mai. Pat. wieder etwas klarer.

9. Mai. Exitus sehr plötzlich, nachdem Pat. vorher noch sein Mittagessen im Bette sitzend eingenommen hatte.

Einer besseren Uebersicht halber möchte ich zum Schlusse die hauptsächlichsten klinisch beobachteten Symptome, wie sie nach und nach sich summirend aufeinander folgten, noch einmal kurz zusammen. fassen :

Psychische Depression, Apathie, leichte Gedächtnissschwäche, heftiger Kopfs chmerz in Stirn- und Nackengegend (Beklopfen des Schädels, besonders am Scheitel, schmerzhaft); leichte Nackenstarre; starkes Schwindelgefühl beim Aufrichten.

Sehschärfestark herabgesetzt (ophthalmoskopisch: Beiderseits exquisite Staungspapille und multiple grosse Retinalhämorrhagien).

Erbrechen. Pulsverlangsamung.

Oefter kurze Anfälle von Bewusstlosigkeit, hie und da anch mit ineonstanten motorischen Reizerscheinungen (beide Arme, linker Facialis, rechter Abducens).

Oefter vorübergebende Obscurationen; Kopfschmerzen, in ihrer Intensität sehr wechselnd; bei besonders heftigem Kopfschmerz deutliche Pulsverlangsamung und Erweiterung der linken Pupille. - Später links Oculomotorius-Parese; links Abducens-Parese. Reflectorische Pupillenstarre. Allmählich völlige Amaurose. Rechts Facialis-Parese.

Incontinentia urinae et alvi.

Zuletzt täglich Anfälle von Bewusstosigkeit ohne motorische Reizerscheinungen (von ca. 10 Minuten langer Dauer). Exitus ganz plötzlich, ohne Agone. 


\section{Diagnose.}

Die allgemeinen, soeben noch einmal kurz zusammengefassten Erscheinungen des langsam, aber stetig (wenn auch unter Schwankungen) wachsenden Hirndrucks liessen auf einen allmählich, aber stetig wachsenden raumbeschränkenden Process schliessen, localisirt an der Basis des Mittelhirns, mit Druck oder auch Uebergreifen auf die oben genannten Nervenstämme.

Bei der klinischen Besprechung des Falles wurde zugleich die Wahrscheinlichkeit eines jenen Process begleitenden Hydrops des III. Ventrikels berührt, als Erklärung für die im Verlauf der Krankheit entstandene beiderseitige totale Amaurose (eingeleitet durch öfters eintretende momentane Obscurationen) durch Druck des Flüssigkeitssackes des erweiterten III. Ventrikels auf das Chiasma oder die Tractus nervorum opticorum.

Nach dem klinischen Verlauf, der auch einen langsamen, stetig fortschreitenden Kräfteverfall des Patienten mit sich brachte, wurde als wahrscheinlichste Form der Erkrankung ein langsam wachsender, maligner Tumor an der Basis des Mittelhirns angenommen, entweder von der Hirnsubstanz selbst, oder von der Schädelbasis, oder von den Hirnhäuten an dieser Stelle ausgehend (das frühzeitige Auftreten von Störungen im Gebiete der NN. optici musste auch die Möglichkeit eines Hypophysis-Tumors erwägen lassen), also ein Gliosarkom oder Sarkom.

Eine luetische Periostitis oder ein Hirngumma konnte wegen Fehlens aller Zeichen überstandener Lues und wegen der Wirkungslosigkeit von länger fortgebranchtem Jodkali (2-3,0 pro die) ausgeschlossen werden, ebenso ein Solitärtuberkel wegen des Alters des Patienten, der starken Hirndruckerscheinungen, des völlig fieberlosen Krankheitsverlaufs und des Mangels irgend welcher anderswo localisirten Erscheinungen von Tuberculose.

Sectionsbefund (Prof. v. Kahlden) 10. Mai 1891.

Schädelknochen auffallend dick, fast keine Diploë.

Dura beiderseits mässig injicirt, zart, an der Innenfläche glatt. $O$ ber flache des Gehirns starkabgeplattet, Sulciverstrichen. Pia besonders längs der Gefässe streifig getrübt. Frische Exsudate in der Pia nirgends zu bemerken. Aus dem Trichter entleert sich bei Herausnahmedes Gehirns wässrige, klare, durchsichtige Flüssigkeit strahlartig in grosser Menge.

An der Oberfläche des Gehirns bemerkt man besonders über der linken Hemisphäre kleine, warzenartige Excrescensen anf der Pia, die stecknadelkopfgross oder kleiner sind und sich mit der Pia zusammen leicht entfernen lassen. Beide Seitenventrikel stark erweitert; Gefässe 
an der Basis beider Ventrikel stark injicirt. Boden der Seitenventrikel glatt. Noch bedeutender zeigt sich nach Durchschneidung des Balkens und Fornix die Erweiterung des dritten Ventrikels, der einen fast wallnussgrossen Hohlraum darstellt. Mittlere Commissur ziemlich breit, in ihrer Form wollerhalten; Marksubstanz der Grossbirnhemisphären mässig fest, beiderseits Herderkrankungen nicht nachzuweisen; centrale Ganglien etwas weicher, ebenfalls ohme Herderkrankungen. Nach binten zu wird die Consistenz des Gehirns äusserst weich; namentlich Pons und noch mehr die Medulla sind fast breiig. Centralkanal des Rückenmarks erscheint im obersten Theil etwas erweitert. Gefässe an der Gehirn-, wie Schädelbasis frei.

An der Vena magna Galeni, Foramen Monroi und Umschlagstellen der Pia nach den Ventrikeln zu nichts Abnormes.

Nach Herausnahme des Räckenmarks bemerkt man einen anffallenden Consistenzunterschied zwischen der oberen und unteren Hälfte. Das Hals- und der obere Theil des Brustmarlues sind breiig we i ch, während alle unteren Partien ziemlich fest sind. In der Arachnoidea sieht man an einzelnen Stellen kleine Kalkplättchen, i w U ebrigen sind die Partien des Rü ckenmarks, namentlich des Hals-, weniger des oberen Brustmarks, deutlich abgeplattet. Die schon vorher durch das Gefühl als erweicht erkannten Partien zeigen auf dem Durchschnitte nur noch undeutliche Structurzeichnungen. Innerhalb des Markes findet sich jedoch eine mit Flüssigkeit gefüllte Höhle, anscheinend mehr nach links als nach rechts sich erstreckend. Weitere Verhältnisse sind wegen der Erweichung makroskopisch nicht zu eruiren.

Im Lendenmark ist die Zeichnung deutlich, die Marksubstanz auch hier deutlich erweicht. In den alleruntersten Partien sind die grauen Hörner stärker geröthet.

Linke Lu nge fast frei von Verwachsungen, die rechte in etwas grösserer Ausdehnung mit dem Rippenfell verwachsen. Herz ziemlich gross, linker Ventrikel etwas erweitert, seine Musculatur 15-17 Mm. breit, braun, von mässig fester Consistenz. In der Aorta kleine atheromatöse Herde, die sich besonders auch auf das Endocard unterhalb der Aortenklappen fortsetzen, während letztere selbst zart sind. Mitralklappe ziemlich stark verdickt. Papillarmuskeln an einzelnen Stellen etwas schwielig.

Beide Lungen ziemlich gross, in der linken findet sich an der Spitze des Oberlappens, etwa in der vorderen Axillarlinie, ein stecknadelkopfgrosser verkalkter Herd. Auf dem Durchschnitt ist das Organ lufthaltig, ziemlich blutreich, jedoch finden sich an der Basis des Unterlappens einige kleinere linsengrosse, stärker geröthete Stellen luftleer; Bronchien mässig weit, Bronchialschleimhaut ziemlich stark geröthet. In der rechten Lunge findet sich ebenfalls etwas nach unten von der Axillarlinie ein stecknadelkopfgrosser, pigmentirter, verkalkter Herd. Durchschnitt, wie links, ziemlich blutreich, durchweg lufthaltig. Bronchien hier weiter als links, mit ziemlich reichlichem Schleim bedeckt.

Milz etwas vergrössert, Pulpa grauroth, Follikel als kleine graue Punkte deutlich zu erkennen, Consistenz weich.

Linke Niere gross, lässt sich aus ibrer Kapsel nur mit zahlreichen Substanzverlusten auslösen. Oberfläche blauroth, Consistenz mässig fest; 
auf dem Durchschnitte erseheint die Rindensubstanz fast ebenso dunkelroth wie die Marksubstanz, so dass beide Theile nicht sehr deutlich von einander abzngrenzen sind. Dabei finden sich in der Rinde an einzelnen Stellen kleine, gelbe Bezirke. Rechte Niere zeigt dieselbe Adhärenz der Kapsel; Durchschnitt auffallend feucht, Rinde hier im Ganzen ebenso dunkel, doch treten in grösserer Ausdehnung als links mehr graue bis grangelbe Partien hervor. Markstrahlen sind als dunkelblaurothe Streifen deutlich zil erkennen.

Die Leber zeigt eine Veränderung des linken Lappens, der durch eine Furche bindegewebiger Art eine Art von Zweitheilung erfahren hat. Organ ist gross, Durchschnitt grauroth, Centren der Acini deutlich zu sehen. Gallenblase frei.

Leichendiagnose: Hydrocephalus internus, Syringomyelie, Bronchitis, Atherom der Aorta, Pleuritis adhaesiva, Staungspapille.

Die Autopsie ergab also nirgends im Gehirn Herderkrankungen, sondern nur einen sehr stark sackförmig ausgedehnten, mit klarer wasserheller Flüssigkeit prall gefüllten III. Ventrikel, der nach unten stark prominirte, so dass die seitlich und vorn von ihm verlaufenden Hirnnerven dadurch theilweise platt gedrü ckt wurden (Chiasma der NN. optici, NN. Oculomotor., Abducens und Facialis); Ventrikelependym völlig normal; Hirnhäute völlig frei, zart und glatt.

Als zufällig fand sich eine ziemlich bedeutende Spaltbildung im Rückenmark, und zwar, was jedoch erst nach Härtung. in Müller'scher Flüssigkeit deutlich wurde, in den beiden Hörnern der grauen Substanz, besonders aber links, von der Medulla oblongata an herabreichend bis zur Mitte des Dorsalmarks.

Indem ich die Localisation und Grössenverhältnisse dieser Spaltbildung an den beigefügten Zeichnungen verschiedener untereinander angelegter Rückenmarksquerschnitte anschaulich zu machen versuche, will ich nun zur Beschreibung des mikroskopischen Untersuchungsergebnisses ïbergehen: ${ }^{1}$ )

Sämmtliche durch die Medulla oblongata gelegten Sehnitte erweisen sich unter dem Mikroskope als völlig den normalen Verhältnissen entsprechend; eine Spaltbildung ist hier nirgends zu entdecken.

Erst in der Höhe der Pyramidenkreuzung gelegte Schnitte lassen die beginnende Spaltbildung erkennen: Hier erblickt man links seitlich von

1) Gehirn und Rückenmark wurden direct in Müller'scher Flüssigkeit fünf Monate gehärtet (die letzten 14 Tage wegen noch nicht genügend bewirkter Härtung im Brütofen); dann in Alkohol; Einbettung in Celloidin; Färbnng, theils mit Hämatoxilin, Alaun-Carmin, theils nach Weigert. 
Pyr.-Kreuzung.

I. D. N.
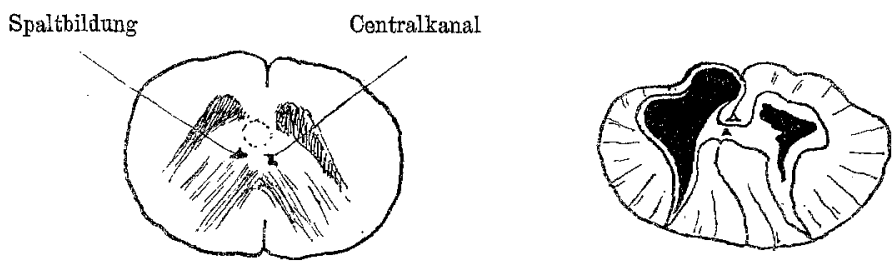

I. C. N.

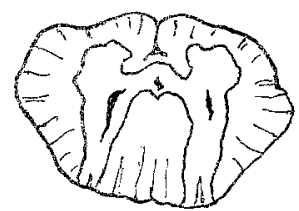

II. C. N.

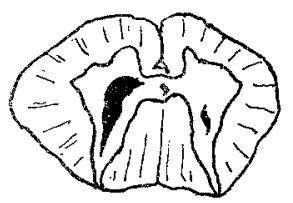

IV. C. N.

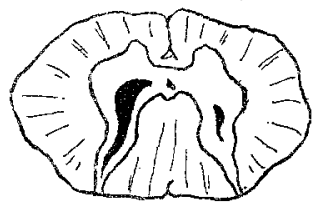

VI. C. N

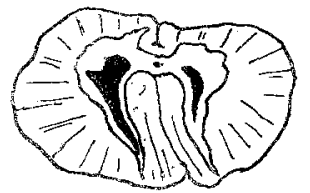

II. D. N.

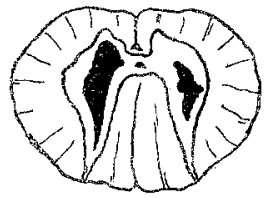

IV. D. N.

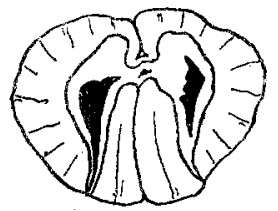

V. D. N.

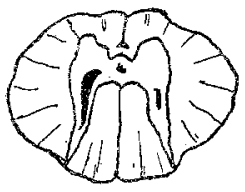

VI. D. N.

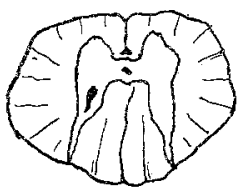

Ruckenmarksquerschnitte, in doppelter Vergrösserung. 
dem etwas nach links verlagerten, allerdings zwar etwas erweiterten und im Querschnitt etwas y-förmig erscheinenden, aber völlig isolirt verlaufenden, mit hohem Cylinderepithel ansgekleideten Centralkanal eine im Querschnitt dreieckige Spaltbildung von sehr kleinem Lumen, die in dem obersten Theile der die Decussatio pyramidum betreffenden Querschnitte nicht mehr zu entdecken ist, also daselbst blind (nach oben) endigt. A n ihrer Wand ist Epithel nirgends zu entdecken, und das den Spalt umgebende Gewebe zeigt nirgends eine durch stärkere Tinction kenntliche Vermehrung von Glia oder Zunahme von Gefässen, Anbäufung von Rundzellen, oder auch irgend welche Zeichen von Erweichungen oder frischeren, resp. älteren Blutungen (wie Pigment u. s. w.). Die in der unmittelbaren Umgebung des Spaltes befindlichen Axencylinder erweisen sich als durch Carmin leuchtend roth tingirt, und nach Weige rt gefärbte Schnitte lassen deutlich deren schwarz gefärbte Markscheiden erkennen. Die rechte Hälfte dieser Querschnitte lässt nirgends eine Spaltbildung, oder auch eventuell nur deren Vorstadinm, eine durch stärkere Tinction sich bemerkbar machende Gliavermehrung erkennen.

In der Höhe des Abgangs des I. Cervicalnerven ist die Spaltbildung in dem linken Hinterhorn schon etwas weiter vorgeschritten, im rechten Hinterhorn beginnt hier ebenfalls eine, im Lumen jedoch wesentlich kleinere Spaltbildung. Centralkanal vielleicht etwas erweitert, an normaler Stellung völlig isolirt von den beiden Spaltbildungen mit wohlerhaltenem Epithel ausgekleidet sichtbar, in seiner Umgebung keine concentrische Bindegewebsverdickung seiner Wand, keine Anhäufung von Rundzellen.

In der Höhe des Abgängs des II. Cervicalnerven Centralkanal von nähnadeldünner Weite an normaler Stelle nachweisbar mit gut erhaltenem, hohem, einschichtigem Oylinderepithel ansgekleidet, in dessen Umgebung keine Vermehrung der Glia oder der Gefässe, auch keine Anhäufung von Rundzellen. Links seitlich von dem Centralkanal innerhalb des Gewebes des linken Hinterhorns eine Spalte von länglicher Dreieckform, deren hinterer, spitzester Winkel bis an die hintere Wurzel reicht, während seine beiden vorderen Ecken in der Höhe des Centralkanals, aber völlig isolirt von demselben liegen; die Wand dieses Spaltes ist glatt, zeigt aber an ihrem Rande keine Andeutung von Gliawuchernng, auch nicht in Gestalt einer Kapselbildung, keine Deiter s'sehen Zellen; vielmehr lassen sich bis unmittelbar an den Spaltrand nach W eig e rt'scher Methode schön schwarz gefärbte Markscheiden an den Nervenfasern des linken Hinterhornes (dessen Gewebe durch den Spalt lediglich etwas auseinandergedrängt erseheint) ebenso wie deutlich tingirte grosse Vorderhornganglienzellen vor ihm und bis dicht an seine vordere Seite herangehend erkennen; auch mit Carmin behandelte Schnitte zeigen einen schön roth gefärbten Axencylinder, während eine stärkere Tinetion an dem Rand der Spaltbildung, als Ausdruck einer Gliawucherung, nirgends zu bemerken ist, ebensowenig wie eine Vermehrung der Gefässe (die im Gegentheil in der Umgebung äusserst spärlich erscheinen), oder Reste von Pigmentablagerung; nu r um die dem Centralkanal am nächsten gelegene Ecke des Spaltes bemerkt man eine ganz düne Schieht von Gliagewebe, erkenubar dureh stärkere Rothfärbung mit Car- 
min, ein zartfaseriges Netzwerkdarstellend, in welchem jedoch Deiters'sche Zellen nicht zu entdecken sind. Im rechten Hinterhorn, ungefähr symmetrisch zu der anderseitigen Spaltbildung gelegen, ebenfalls eine, jedoch ca. 4 mal kleinere Spalte, deren Umgebung der eben beschriebenen völlig gleicht, nur dass das begrenzende Hinterhorngewebe hier theilweise etwas fetzig auseinandergedrängt erscheint durch kleine in dasselbe einspringende Spältchen; auch hier nirgends eine Andeutung von den Spalt begrenzender Neurogliaschicht. An der inneren Wand der Spalten, weder links noch rechts, irgend welche Reste von Epithel zu entdecken. Die Pia stellt überall ein zartfaseriges, nirgends verdicktes Gewebe dar, das nirgends eine Anhäufung von Rundzellen zeigt, und dessen Gefässe eine zarte Wand haben und nirgends obliterirt erscheinen.

In der weissen Substanz erscheinen die Axencylinder mit Carmin schön roth gefärbt, ebenso wie die Markscheiden schön schwarzbraun (nach Weigert gefärbt).

In der Höhe des Abgangs des VI. Cervicalnerven: Der nicht erweiterte Centralkanal isolirt an normaler Stelle mit wohlerhaltenem Epithel ausgekleidet; seine Umgebang zeigt nirgends Gliawucherung. Links seitlich von demselben, aber getrennt von ihm durch normales Gewebe eine Spalte von nahezu dreieckiger Gestalt, fast das ganze linke Hinterhorn einnehmend, von etwas unregelmässiger, fetziger Begrenzung. Das Gewebe des linken Hinterhorns erscheint hierdurch etwas zur Seite und zusammengedrängt, doch sind bis unmittelbar an die Spaltwand heran iberall schwarz gefärbte (W eigert) Markscheiden zu erkennen. Die nach vorn von ihm liegenden grossen Vorderhornganglienzellen erscheinen an Zahl vielleicht etwas vermindert, aber schön tingirt, und der stellenweise aus ihnen hervorgehende Axencylinder mit Carmin deutlich roth gefärbt; an der Wand der Spalte nirgends Gliawucherung zu erkennen, ebenso auch nirgends Epithelreste. Rechts seitlich, symmetrisch gelegen zn der links, eine ca. 3 mal kleinere Spalte, deren Begrenzung und Umgebung sich genau wie links verhält. Pia ïberall zart, nirgends verdickt, ohne Kernwucherung oder Gefässveränderung.

In der Höhezwischen VII. Cervical-und I. Dorsalnerven Abgange: Centralkanal an normaler Stelle, isolirt, obliterirt; keine Gliawucherung um denselben. Indem linken Horn der grauen Rückenmarkssubstanz heranreichend, so dass nach vorn bis unmittelbar an die weisse Substanz eine grosse Spaltbild ung dieselbe nach innen und vorn gedrängt erscheint, während eine graue Substanz hier nicht mehr zu erkennen ist; von der Höhe des Centralkanals aus nach hinten zu erscheint die graue Substanz durch Compression nur sehr verschmälert bis zur hinteren Wurzel, bis wohin sich auch die Spaltbildung erstreckt; die dreieckige Spalte zeigt um die vordere, in nere Ecke eine dünne Schicht von leicht welligem Aussehen, gebildet von einem zartfaserigen Bindegewebe mit spärlichen Kernen und sehr spärlicher Gefässentwicklung, aber ohne Deiters'sche Zellen (A ndeutung einer Kapselbildung). Diese Faserschicht wird nach vorn direet begrenzt von einer durch Compression etwas verschmälerten weissen Substanz, innerhalb welcher aber die Axencylinder mit Carmin schön roth gefärbt erscheinen, während die nach Weigert gefärbten Schnitte ein vielleicht etwas geringeres, mehr gelbliches Tinctionsvermögen der Markscheiden 
erkennen lassen. Die grossen Vorderhornganglienzellen fehlen hier natürlich vollständig, dagegen bemerkt man an dem nach aussen gedrängten linken Seitenhorn noch einige gut tingirte multipolare Ganglienzellen; in den hinteren beiden Dritteln des Spaltes erscheint die graue Substanz nur verschmälert, aber ihre bis unmittelbar an die Spalte heranreichenden Nervenfasern zeigen sich nach Weigert gut differenzirt, ebenso wie die Nervenfasern in den linken Seiten- und Hintersträngen normal tingirte Axencylinder (Carmin) und Markscheiden (W eig e rt) erkennen lassen. Im rechten Horn der grauen Rückenmarkssubstanz ebenfalls eine, aber circa 2 mal kleinere Spaltbildung, deren Begrenzung unregelmässig etwas fetzig erscheint, und die nirgends von einer bindegewebigen Schicht umgeben ist, so dass die Nervenfasern und Zellen der grauen Substanz bis unmittelbar an die Höhle heranreichen, überall gut tingirt; die grossen Vorderhornganglienzellen nach innen und vorn gedrängt, jedoch mit deutlich hervortretendem Kern; nur erscheinen sie an Zahl vielleicht etwas vermindert; die umliegende weisse Substanz lässt nirgends eine Veränderung erkennen. Beide Spalten zeigen an keiner Stelle ihrer. Wand Epithel oder dessen Reste. Die Pia ist nirgends verdickt und zeigt auch in ihren Einstülpungen keine Anhäufung von Rundzellen.

In der Höhe des Austritts des IV. Dorsalnerven: Centralkanal an normaler Stelle mit wohlerhaltenem Epithel ausgekleidet, nicht erweitert, völlig isolirt; in seiner Umgebung keine Gliavermehrung; durch eine Schicht völlig normalen Gewebes getrennt von ihm verläuft im linken Horn der grauen Rückenmarkssubstanz eine im Querschnitt dreieckige Spaltbildung mit glatter Wandung, an deren vorderer innerer Ecke kapselartig eine dünne Schicht von feinfaserigen Gewebe sich zeigt (ohne Vermehrung der Gefässe oder Kerne), in welcher Deiters'sche Zellen nicht bemerkt werden, die aber mit Carmin sich stark tingirt; an allen übrigen Theilen der Höhle reicht das Gewebe der grauen Substanz bis unmittelbar an die Höhle heran und zeigt gut differenzirte Nervenfasern and Kerne. Die grossen Vorderhornganglienzellen erscheinen etwas nach vorn und zusammengedrängt, an Zahl vielleicht etwas vermindert, aber von gutem Tinctionsvermögen, ebenso wie ihre Axencylinder. Rechts symmetrisch zu links gelegene ca. $1 / 2$ so weite Spalte mit etwas fetziger Wand, so dass an vielen Stellen die hier überall gut tingirten Nervenfasern in die Höhle hineinragen, an den anderen Stellen bis unmittelbar an die Höhle reichen. In der weissen Substanz überall Markscheiden und Azencylinder deutlich gut tingirt nachweisbar. Die innere Wand der Höhlen zeigt nirgends Epithelauskleidung. Pia nirgends verdickt, keine Kernvermehrung, keine Gefässobliteration.

Die Höhlen nehmen nun nach abwärts in ihrem Lumen immer mehr ab, die Höhle im rechten Horn endigt in der Höhe des $V$. Dorsalnervenabgangs, die Höhlenbildung im linken Horn in der Höhe des VI. Dorsalnervenabgangs; das Gewebe zeigt auf diesen Querschnitten dieselben Verhältnisse wie oben, die graue Substanz erscheint nur weniger verdrängt, ihre Ganglienzellen und Nervenfasern gut tingirt, die weisse Substanz zeigt stark gefärbte Axencylinder und gut differenzirte Markscheiden. Pia auch hier nirgends verdickt; eine Wucherung der Gliagefässe oder Kerne in der Umgebung des Spalts nicht nachweisbar, ebenso auch nirgends Epi- 
thelreste an der inneren Spaltwand. Der Centralkanal verläuft isolirt an normaler Stelle mit wohl erhaltenem Cylinderepithel ausgekleidet.

Von der Höhe des VI. Dorsalnervenabgangs nach abwärts befindet sich weder makroskopisch, noch mikroskopisch irgend eine Abnormität in den Rückenmarksquerschnitten.

Es folge nun noch die Beschreibung des mikroskopischen Untersuchungsergebnisses der Augen und Optici:1)

Opticusscheide schlaff, runzelig, durch Flüssigkeit (besonders in der Nähe des Bulbus) theilweise bis zu $2 \mathrm{Mm}$. von dem Nervenstamm abgedrängt. Die transversalen Bindegewebsfasern der Lamina cribrosa etwas bogenförmig vorspringend. Papille erheblich geschwollen, fast $2 \mathrm{Mm}$. den Chorioidealrand ïberragend mit unregelmässiger buckliger Verdrängung der benachbarten Retinalpartien, grösstentheils durch Blutextravasate. In der Papille ebenfalls Blutextravasate und spärlich Blutpigment. Im N. opticus von der Lamina cribrosa an, centralwärts reichlicher werdend, Fettkörnchen. Die Markscheiden der Opticusfasern zum grössten Theil in Degeneration begriffen. Die Axencylinder nach Flemming nicht zu beurtheilen. ${ }^{2}$ )

Um nun in erster Linie auf die Pathogenese unserer Spaltbildung einzugehen, so käme als die häufigste Ursache der Syringo. myelie zunächst in Betracht

I. eine Gliomatos e mit secundärem centralen Zerfall. Diese glaube ich aus folgenden Grïnden ausschliessen zu können: Es wäre auffallend, dass eine Gliomatose sich fast genau symmetrisch, gegenseitig isolirt, nur auf die beiden Hörner der grauen Substanz beschränken sollte, in ihrem ganzen Verlauf vorn entsprechend den breiten Vorderhörnern breiter, hinten entsprechend den schmalen Hinterhörnern schmäler, nirgends auch in die weisse Substanz übergehen, nirgends mit der anderen Seite in Verbindung treten, und nirgends den Centralkanal in Mitleidenschaft ziehen sollte. Ausserdem finden wir in obiger Untersuchung absolut keinen Anhaltspunkt dafiir, dass wir es mit einer Gliomatose zu thun hätten; das Fehlen jeder stärkeren Wucherung von Gliagewebe schliesst echte Gliomatose aus; denn die dünne Schicht Glia, die sich an einigen Stellen, jedoch nur in ganz geringer Breitenausdehnung, und zwar nur an einer Ecke der linken Spalte findet, kann wohl nicht als eine primäre Gliomatose angesehen werden, sondern muss vielmehr als eine secundäre, durch die Spaltbildung hervorgerufene leichte Bindegewebswucherung, besonders nach dem Centralkanal zu, angesprochen

1) Bulbus mit daranhängendem Opticus in Flemming'sche Lösung; später Härtung in Alkohol; Einbettung in Celloidin, Färbung in Saffranin.

2) Eine eingehendere Untersuchung der Bulbi und Optici wird von Herrn Dr. Ströbe, damals Assistenten am pathologischen Institut in Freiburg i. B.; erfolgen, und deren Resultat in Zieg ler's "Beiträgen" mitgetheilt werden. 
werden, wie sich solche bindegewebigen Wände (Kapseln) ja auch an anderen Organen, z. B. bei den Blutcysten im Gehirn, oder bei den durch Erweichung entstandenen Cysten in Fibromen nachweisen lassen, als Ausdruck eines spontanen Ausheilungs- oder wenigstens Einschränkungsversuchs, ein Kampf des normalen Gewebes gegen pathologische Vorgänge. Ausserdem wäre die Spaltbildung rechts hierdurch gar nicht zu erklären, denn wir finden rechts auch nicht die geringste Andeutung von Wucherung der Glia. Das Fehlen der Deiters'schen Zellen in dem sehr spärlich vorhandenen Bindegewebe spricht wohl ebenfalls gegen eine primäre Gliomatose.

II. Wollten wir auf die Langhan s'sche ${ }^{1}$ ) Theorie zuriickgreifen, dass bei Hirntumoren durch Circulationsstörung Spaltbildungen im Rückenmark entstehen können, so sollten wir doch eher eine Erweiterung des Centralkanals erwarten, als das Entstehen nener Höblen in den Hörnern des Rückenmarks. Ausserdem ist dafür, dass in unserem Falle jemals im Rïckenmark Circulationsstörungen bestanden hätten, kein Beweis zu erbringen, da weder erweiterte Blutgefässe, noch Blutungen, noch Pigment als deren Rest sich nachweisen lassen.

III. Könnte man an eine Spaltbildung myelitischer oder hämorrhagischer Genese denken. Hierfür findet sich jedoch nicht der geringste Anhaltspunkt. Denn es fehlt eine Vermehrung oder Erweiterung der Blutgefässe in der Umgebung der Spaltbildung, sowie der Rest einer Hämorrhagie, wie Blatpigment; auch von myelitischen Veränderungen finden wir nirgends etwas in unserem Falle, abgesehen davon, dass die Symmetrie der Spaltbildungen und ihr Beschränktsein auf die Hörner der grauen Substanz eine Myelitis oder Hämorrhagie mit Sicherheit ausschliessen lassen.

IV. Wäre an eine Höhlenbildung infolge von regressivem Gewebszerfall zu denken. Aber auch hierfür fehlen jegliche Anhaltspunkte, und würde sich namentlich die Symmetrie der Höhlen und ihr Beschränktsein auf die Hörner gar nicht erklären lassen.

Der bisherigen Betrachtung folgend werden wir immer mehr zu der Annahme gedrängt, dass wir es mit einer congenitalen Anlage zu thun haben, und es kommen hierbei wieder mehrere Möglichkeiten in Betracht. Wir könnten unsere Spaltbildungen

1. als Productevon Ausstülpungen des Centralkanals (Divertikelbildung) mit secundärer Abschnürung ansprechen. Hiergegen spricht jedoch das völlige Fehlen jeglicher Communication zwischen dem eigentlichen Centralkanal und dieser Höhle an irgend

1) Virchow's Archiv. Bd. 85. S. 1 ff. 
einer Stelle (soweit sich dies beurtheilen lässt; denn wenn auch diese vielleicht der Beobachtung entgangen sein könnte, so müsste doch irgendwo eine Erweiterung des Centralkanals bestehen), ferner das Fehlen von Epithel oder dessen Resten an irgend einer Stelle der Wand der Höblen. Allerdings lebrt die Erfabrung, dass das die Wand solcher Hohlräume auskleidende Epithel häufig untergeht, sei es, dass die in der Höhle enthaltende Flüssigkeit durch ihre Bewegung, sei es durch ihren vermehrten Druck solehes bewirkt. (Auch ist nicht anszuschliessen, dass durch die postmortale Behandlung der Präparate einzelne Epitheltheile ausgefallen sein könnten, obgleich man wohl annehmen darf, dass bei der jetzigen Celloidinbehandlung sich das Epithel doch conserviren lassen sollte, zumal da es ja auch im Centralkanal tiberall noch vorhanden ist).

2. Könnten wir es mit einer congenitalen Anlage vondrei Rü ckenmarkskanälen zu thun haben, von denen sich die beiden seitlichen aus uns unbekannten Gründen durch Zunahme der in ihnen enthaltenen Flüssigkeit erweitert hätten. Hiergegen aber spricht erstens der Mangel jeglichen Epithels an den Wänden der Spalten (was jedoch nach den oben angeführten Grïnden kein absoluter Gegenbeweis ist) und zweitens der nicht erweiterte, an normaler Stelle gelegene mittlere Centralkanal, der doch sicher am meisten Ursache gehabt hätte, sich za erweitern, besonders wenn wir nach der Lang hans'schen Theorie annehmen, dass durch Raum beschränkende Processe in der hinteren Schädelgrube (z.B.Hydrocephalus internus) secundär durch Anstaungen eine Erweiterung des Centralkanals entstände. ${ }^{1}$ )

So bliebe denn als letzte, wahrscheinlichste Erklärung für die Spaltbildung in unserem Fall nur übrig, anzunehmen, dass wir es mit congenital präformirten, symmetrisch in der oberen Hälfte der Hörner der grauen Rïckenmarkssubstanz gelegenen Höhlen za thun haben, deren embryologische Entstehung allerdings schwer zu erklären sein dürfte, und wofür wir anatomische Beweise bisher nicht zu erbringen vermögen. Dass wir es mit einer congenitalen Missbildung zu thun haben, hierfïr spricht ja auch die Erfahrung, dass Missbildungen in Gehirn und Rüekenmark häufig combinirt vorkommen, und wir ja den Hydrocephalus internus in unserem Fall auch als congenital anzusehen berechtigt sind, wie wir später zu beweisen versuchen werden.

1) Wir können somit auch die Langhans'sche Theorie auf unseren Fall gar nicht anwenden, also den Hydrocephalus internus in k eine n ätiologischen Zusammenhang bringen mit der etwa durch ihn secundär bewirkten Spaltbildung im Rückenmark. 
Das Fehlen jeglicher spinaler Symptome intra vitam wird ebenfalls am besten durch die Annahme einer congenitalen Missbildung erklärt, und die an einzelnen Stellen des linken Vorderhorns scheinbar verringerte Zahl der grossen Vorderhornganglienzellen - (das einzig Pathologische, was wir mikroskopisch am Rückenmarksgewebe nachweisen konnten) - berechtigt uns nicht zur Annahme, dieselben seien in Wahrheit an Zahl vermindert (z. B. durch Druckatrophie), da wir ja nur die Zahl derselben, einer Ebene nach, beurtheilen können.

Unsere Ansicht in der Erklärung dieses immerhin äusserst seltenen Falles geht also dahin, dass wir es mit einem congenitalen Hydrocephalus internus geringen Grades (vgl. hierzu S.129), complicirt mit eongenitaler Spaltbildung in den grauen Hörnern der oberen Hälfte des Rückenmarks zu thun haben, von denen der erstere aus uns unbekannten Gründen erst im höheren Lebensalter plötzlich unter Tumorerscheinungen so zunahm, dass er den Tod herbeifuhrte, während die Spaltbildungen im Rückenmark wohl intra vitam nur sehr langsam und symptomlos zugenommen haben, was auch daraus hervorgeht, dass wir in dem Rückenmark lediglich Verdrängungserseheinungen leich ten Grades, aber keinen Verlust von Gewebe finden.

Die Publication dieses Falles in extenso scheint uns, wie gesagt, nach zwei Richtungen hin von besonderem Interesse; und zwar 1. wegen des äusserst selten beobachteten Vorkommens eines i di op a thischen Hydrocephalus des Erwachsenen (mit typischen Hirntumorsymptomen) und 2. dessen geradezu einzig dastehender Complication mit Syringomyelie. - Beim Durchsuchen der auf Punkt 1 bezïglichen Literatur konnte ich im Ganzen nur drei Fälle von zweifellos idiopathischem Hydrocephalus internus der Erwachsenen finden, von denen zwei, wie der unsere, mit typischen Hirntumorsymptomen verliefen, der dritte unter der Form einer Meningitis. Und beim Durchsuchen der an unserer Klinik etwa beobachteten ähnlichen Fälle wurde ich noch auf einen vierten Fall aufmerksam, der ebenfalls unter den Symptomen eines Hirntumors verlief, sich aber post mortem gleichfalls als idiopathischer Hydrocephalus internus erwies.

Es mögen hier diese vier Fälle im Auszug mitgetheilt werden. Der erste Fall wird von Anuske') publicirt.

1) Die Neuritis optica bei Tumor cerebri. Gräfe's Archiv f. Ophthalmologie. Berlin 1873. Bd. XIX. Abth. III. Fall VI. S. 260. 
An amnese: August Weiss, 31 Jahre, Arbeiter, aus gesunder Familie, bis zur jetzigen Krankheit stets gesund; seit ca. 3-4 Monaten Kopfschmerzen und Schwindel; gleichzeitig rapide Abnahme des Sehvermögens bis zur totalen Erblindung; seit 3 Wochen Lähmung und Gefühllosigkeit der rechten Gesichtshälfte.

Status (am 5. Juli 1872 Aufnahme in die Klinik; am 8. und 10. Juli 2 mal Anfälle von tiefem Coma). Sensorium und Psyche frei, Sprache normal, geringe beiderseitige Abducens-Parese. Hypästhesie auf der rechten Gesichtshälfte; beiderseits totale Amaurose. Beiderseits Neuritis optica mit sehr starker Papillenschwellung und zahlreichen kleineren und grösseren Apoplexien auf $\mathrm{Pa-}$ pillen und Retina. - Pupillen sehr weit, träge reagirend. Starker Schwindel nnd Schwanken beim Aufrichten; Gehen hierdurch unmöglich. Puls 76; kein Fieber. - Kein Erbrechen. - Am 23. und 30. Juli 2 mal $1 / 2$ stündiger Comaanfall mit epileptiformen Zuckungen in den Extremitäten; Kopf- und Genickschmerz nachher.

1. August. Linksseitige Facialis-Parese. Schlingbeschwerden. Exitus.

6. August. Kurzer Anfall von Coma Morgens. Abends plötzlich

Sectionsprotokoll: Gehirnwindungen abgeplattet. „An der Basis erscheint dicht hinter dem Chiasma und nach hinten bis an die Crura cerebri hinreichend eine prall gefüllte dünnwandige Blase, ebenso beiderseits zwischen der hinteren Fläche des Pons, des Cerebellum und der Medulla oblongata je eine pflaumengrosse pralle Blase.“

Die Wand der Blasen wird von Pia allein gebildet, deren Höhlen sich in den 4. Ventrikel hinein fortsetzen; Hydrops aller Ventrikel. Aquaeductus Sylvii sehr weit, Commissura mollis geschwunden; Boden des 4. Ventrikels plattgedrückt, ebenso alle von der Hinterfläche des Pons, sowie vom Anfangstheil der Medulla oblongata abgehenden Nervenstämme. „Die beträchtliche Menge Flüssigkeit hat dieselbe klare wasserbelle Beschaffenheit, wie die aus den Blasen entleerte." Ependym der Ventrikel durchweg glatt und nirgends verdickt; die hintere Partie des Tractus opticus abgeplattet. Pia ohne irgend welche pathologischen Veränderungen.

Wernicke ${ }^{1}$ ), der dieses Falles Erwähnung that, fasst ihn „un zweifelhaft als chronische Meningitis a uf", obschon in dem Sectionsprotokoll eine Erklärung hierfür unserer Ansicht nach nicht zu finden ist. An uske (1. c.) knüpft an seinen Fall noch folgende epikritische Bemerkungen: Er glaubt, ein intracranieller Tumor hätte mit grosser Wahrscheinlichkeit ausgeschlossen und die richtige Diagnose trotz der Seltenheit des Falles gestellt werden können -; zu diesem Ausspruch hält er sich durch folgende Erwägungen berechtigt: „Patient ist in einem Zeitraum von 3 Monaten total erblindet, und der Augenspiegel nöthigt, hievon die Ursache in die Schädelhöhle zu verlegen. Die auf beiden Augen gleichmässige, rapid

1) Lehrbuch der Gehirnkrankheiten. Berlin 1883. Bd. III. S. 529. 
eingetretene quantitative Erblindung ist bei Annahme eines Tumors wohl nur durch directen D ruck desselben auf das Chiasma oder die Nervi optici zu erklären. Andererseits lehrt die Erfabrung, dass bei unmittelbarer Compression der genannten Leitungsapparate entweder ein schneller Uebergang aus Neuritis in weisse Atrophie statthat, oder die letztere gar primär (was noch nicht bewiesen ist) eintritt. Statt dessen finden wir in dem vorliegenden Falle erhebliche Papillenscbwellungen und in solcher Ausdehnung nie beobachtete Stauungsphänomene. Die schnelle Entwicklung der vollständigen Amaurose bei den ausgeprägtesten Staunngserscheinungen in Opticus und Retina erfordern eine schnelle, hohe und anhaltende Druckzunahme in der Schädelhöhle, die ein acuter Hydrops ventricolorum sicher herbeiführen kann, während ein Tumor von so rapidem Wachsthum bei fast vollständigem Mangel von Herdsymptomen ein ebenso seltenes Vorkommniss als die jähe Entwicklung eines Hydrops ventricolorum infolge einer Neubildung sein dürfte." - Hierauf könnte man jedoch erwidern, dass ein idiopathischer Hydrocephalus internus (wie ihn der oben citirte Fall zeigt) ein doch sicherlich noch weit selteneres Vorkommniss bildet, und dass es daher immer noch näher liegt, eine $\mathrm{Neubildung}$ in cerebro anzunehmen, die entweder direct oder durch den begleitenden Hydrops der Ventrikel (der, wie ja aus der Anuske'schen Arbeit hervorgeht, in einzelnen seiner Fälle oft ein „eolossaler" sein kann; siehe 1. c., S. 216-229) auf die Nachbarschaft drüekt und so mehr oder weniger ausgesprochene Reiz- oder Ausfallserscheinungen hervorruft. Ferner ist anch nicht zu vergessen, 1. dass eine nicht allzugeringe Zahl von sogar recht umfänglichen Hirntumoren je nach ibrem Sitze fast ohne Herderscheinungen verlaufen, und 2. dass z. B. Tumoren des Kleinhirns starke Staungspapille mit frühzeitiger Erblindung ${ }^{1}$ ) dadurch veranlassen, dass der Boden des 3. Ventrikels blasenförmig nach unten vorgetrieben das darunterliegende Chiasma, resp. die Tractus comprimirt. Ausserdem kommt gerade bei idiopathischem Hydrocephalus internus durch den Druck des vorgetriebenen Bodens des 3 . Ventrikels primäre Atrophie der Papillen vor. ${ }^{2}$ Vergleiche hierzu auch Wernicke und Leber. ${ }^{3}$ )

Der zweite Parallelfall von idiopathischem Hydrocephalus internus

1) Türck, Ueber Compression und den Ursprung des Sehnerven. Zeitschr. Wiener Aerzte. VIII. Jahrg. II. Bd. S. 299. - Wernicke (1. c.). S. 296.

2) Bouchut, Gaz. des hôp. 44. 45. 1872.

3) Wernicke (1. c.). S. 298 und 299. - Leber, Gräfe-Sämisch's Handbuch der gesammten Augenheilkunde. Leipzig 1877. V. Bd. S. 842. 
der Erwachsenen (ebenfalls mit Tumorsymptomen) ist wiederum an unserer Klinik beobachtet und möge, da noch nicht publicirt, hier etwas ausfïhrlicher mitgetheilt werden:

Anamnese: AnnaRieter, 34 Jahre alt, verheirathet, will früher stets gesund gewesen sein. Eltern und 6 Geschwister leben und sind gesund. Menstruirt mit 19 Jahren, stets unregelmässig, aber ohne Beschwerden; seit der Geburt ihres 3. Kindes (Kinder leben und sind gesund; keine Aborte und keine Frühgeburten) vor einem Jahre Menses sistirt (protrahirte Lactation). - Vor 2 Jahren plötzlich reissender Schmerz im Hinterkopf und öfteres Erbrechen, das auch die folgenden Tage noch fortbestand; danach habe sie öfter Ohrensausen und Schwindelanfälle gehabt, sei öfter bei der Feldarbeit bew usstlos umgefallen und ca. 1/2 Stunde lang bewusstlos geblieben. Die Sehkraft habe nicht abgenommen, aber minde und matt fühle sie sich stets seit diesen 2 Jahren. Vor 9 Wochen fingen wieder stärkere Kopfschmerzen an, und die Schwindelanfälle stellten sich aufs Nene ein, beide stärker als früher, so dass Pat. oft vor Schmerzen habe ,hinausschreien" müssen; beim Gehen sei sie sofort schwindlig geworden, und das Ohrensausen sei wieder aufgetreten; ebenso habe sich wiederum Erbrechen eingestellt; seit 6 Wochen seien auch öfter ca. 1 Stunde andauernde Schwindelanfälle mit Zuckungen, „aber nur in den Augäpfeln", eingetreten. - Die Psyche und das Sensorium seien bis vor Kurzem ganz normal gewesen.

Am 24. August 1889 Eintritt in die hiesige medicinische Klinik.

A ufnahms-Status: Guter Ernährungszustand; starke Demenz (weiss weder ihr Alter, noch die laufende Jahreszahl, dagegen ist Pat. über ilıre Umgebung richtig orientirt); Antworten erfolgen langsam; keine Sprachstörungen, Zunge (keine Atrophie, kein Tremor) wird gerade herausgestreckt; bei stärkeren mimischen Bewegungen fällt eine $\mathrm{schwächere}$ Innervation der linken nnteren Gesichtshälfte auf, doch ist die linke Nasolabialfalte dentlich ausgeprägt. Kein Strabismus; Pat. folgt einem vorgehaltenen Gegenstand mit den Augen nach allen Seiten, doch ist die Seitwärtsbewegung der Bulbi nach links entschieden mit grösserer Anstrengung verbunden, als die nach rechts, und vermag Pat. nur ganz kurze Zeit die Augen stark nach links gewendetzu halten; kein Nystagmus. Der Gang ist breitspurig, schwankend, etwas spastisch; Kopf und Rumpf werdendabei auffallend zur üekgebeugt gehalten. Starkes Schwindelgefüh $l$ beim Verlassen der horizontalen Lage; Motilität der Extremitäten nicht beeinträchtigt. Händedruck links ein wenig schwächer als rechts; passive Beweglichkeit in Armen und Beinen nicht wesentlich behindert; Sebnenreflexe überall lebhaft. Kopf nach allen Seiten frei beweglich, passive Beugung nach vorn frei, nicht schmerzhaft; Beklopfen des Kopfes verursacht nirgends Schmerzen; Nachtschweisse; In continentia urinae et alvi. Residuen von Lues nicht nachweisbar. Im Harn nichts Abnormes.

Therapie: Jodkali 3 mal täglich 0,5 . 
Ophthalmoskopie: Pupillen mittelweit, beiderseits gleich, deutlich reagirend. Rechte $\mathrm{Papille}$ zeigt leicht verwaschene Contouren und ziemlich starke venöse Stauungen; sonst Venen im Augenhintergrund erweitert und geschlängelt. Links der gleiche Befund, nur die Umrandung der Papille eine schärfere.

Am 29. August. Pat. fühlt sich wohler, schwindelfrei, erscheint psychisch etwas lebhafter, antwortet weniger zögernd.

Am 31. August. Pat. ist Morgens früh in einem nicht beobachteten Anfall ans dem Bett gefallen.

10 Uhr Morgens. Anfall im Bett von ca. 3 Minuten Dauer: Bewusstlosigkeit, Erblassen, incoordinirte Bulbusstellungen (linker Bulbus nach oben, rechter nach innen und unten). Pupillen mittelweit, nicht different, reagirend; leichter Nystagmus. In den Extremitäten keine Convulsionen; tonischer Krampf im rechten Facialis. Stöhnen. Puls langsam, 60. Extremitäten kühl, nachher kalter Schweiss; auf der Brast vorübergehend starke Hautröthung. - Nach dem Anfall beide Pupillen dauernd erweitert, aber nicht reactionslos. Einmal Erbrechen; danach Pat. ganz bei Bewusstsein, nur sieht sie blass und collabirt aus. - Nachmittags noch $2 \mathrm{mal}$ ähnliche Anfälle mit lautem Stöhnen. $6 \mathrm{Uhr}$ Abends beim Umbetten plötzlich wieder Bewusstseinsverlust; Extremitäten tonisch gestreckt, Athmung Secunden lang stockend; Puls 150, sehr klein; starke Röthung des Gesichts und der oberen Rumpfpartien; die geröthete Haut fühlt sich aber kühl an; die gleichfalls stockende Respiration kommt nicht wieder in Gang, der Puls wird allmählich langsamer, bleibt bei völlig sistirter Athmung noch ca. 8 Minuten regelmässig und erlischt alsdann, allmählich immer flatternder werdend. Kurz nach 6 Uhr Abends Exitus. - Die A ntopsie ergab: Ziemlich hochgradigen Hydrocephalus internus, besondersdes 3. Ventrikels, der blasenartig nach unten vorgewölbt erscheint; sonstige Veränderungen an dem Gehirn fehlen. An den inneren Organen, ausser einer acuten Stauungsniere, die wohl nur durch die Convulsionen bedingt war, da vorher Albuminurie nicht bestand, nichts Abnormes. (Ein ausführliches Sectionsprotokoll ist leider damals nicht aufgenommen worden, da die Section wegen Reparaturen im Sectionssaal in einer entfernt liegenden Leichenhalle gemacht werden musste.)

Hier wurde ebenfalls nach Anamnese und klinisch beobachtetem Verlauf die Wahrscheinlichkeitsdiagnose auf "Tumor cerebri" gestellt nach den intra vitam beobachteten Erscheinungen, die hier noch einmal kurz zusammengefasst werden sollen: Schwindel, Kopfschmerzen, Erbrechen, öftere Anfälle von Bewusstlosigkeit, meist nur mit convulsivischen Zuckungen der Augenmuskeln, gesteigerte Reflexe, keine Spasmen. Später Parese des linken Facialis, linken Abducens und linken Arms; keine Nackenstarre; ophthalmoskopisch: beginnende Stauungspapille, besonders rechts. - Als Erklärung für all diese Erscheinungen fand sich aber, wie hier gesagt, nur ein starker Hydrops aller Ventrikel (besonders des dritten) bei intactem Ventrikel- 
Ependym und normaler zarter Pia; nirgends in cerebro Herderkrankungen.

Der dritte Fall wird von 0 ppenheim ${ }^{\prime}$ mitgetheilt:

Anamnese: 22 jährige Arbeiterin, die früher stets gesund war; vor 8 Jahren Beginn der jetzigen Erkrankung mit Kopfschmerz, Schwindel, Erbrechen, Ohrensausen, Parästhesien und Schwäche in den Beinen. Im October 1882 Entbindung; danach Verschlimmerung der genanten Symptome und Verminderung der Sehkraft, so dass sie schon nach 6 Wochen auf dem linken Auge nur noch schwachen Lichtschimmer hatte. Im October 1883 zweite Entbindung; daranf Schwäche in den Armen, unwillkürliche Zuckungen in den Beinen und im rechten Arm. Pat. wurde vom 17. December 1883 bis 29. Juli 1884 und dann vom 10. Januar 1888 bis zu ihrem Tode am 29. Juni 1888 auf der Nervenklinik der Berliner Charité behandelt. Bei der ersten Aufnahme 1884 zeigte sich die Pat. geistig beschränkt, leichte Protrusio bulbi. Pupillen weit, aber reagirend, beiderseits Neuritis optica, bitemporale Hemian. opsie. ${ }^{2)}$ Puls beschleunigt, zeitweise unregelmässig; Zittern der Arme; Ataxie und Schwäche im rechten Arm. Im rechten Bein leichte Spasmen und dentlicheSchwäche; lebhafter Patellarreflex, kein Fussclonus. Zeitweise Kopfschmerz, Erbrechen, Heisshunger und Durst; später Ohrensausen, Abnahmedes Hörvermögens und Verlnst des Geruchvermögens.

Diagnose: Tumor cerebri. - Nachdem Pat. das Krankenhaus verlassen, blieb sie bis Mai 1887, d. h. bis zu ihrer 3. Schwangerschaft, arbeitsfähig. Im Juni 1887 mehrfach Uebelkeit und Erbrechen. Seit December 1887 heftige K opf- und Nackenschmerzen, zunehmende Schwäche in den Beinen, besonders rechts, Schwindelgefühl, Abnahme der Sehkraft. 2. Aufnahme in die Charité im Januar 1888. - Ophthalmoskopie wie oben. Deutlicher Nystagmus und Beschränkung der Seitwärtsbewegungen der Augen. Nackenmusculatur gespannt; Bewegungen des Kopfes nach hinten behindert; vollständige Anosmie. Zittern in Armen und Beinen. Im März 1888 Entbindung von einem gesunden Mädchen. End e Juni Lähmung der Beine und Harnverhaltung; starke Pulsbeschleunigung, 144. Stärkere Prominenz der Bulbi. Am 28. Juni Zunahme der Schwäche der Arme. Am 29. Juni 1888 Tod.

Section. 3. Ventrikel ausserordentlich starkblasenartig nach unten vorgetrieben. Chiasma N. opt. und Glandula pituitaria stark comprimirt und abgeplattet. Alle Ventrikel stark erweitert, am meisten der dritte and das Infundibulum. Erweiterung des obersten Theils des Centralkanals des Rückenmarks.

1) Ueber einen Fall von erworbenem idiopathischem Hydrocephalus internus. Charité-Annalen. Jahrgang XV. 1890. S. 309.

2) Nach Schweiger (Gräfe's Archiv. XXII. Bd. III. S. 313) durch Druck einer Geschwulst auf die Fasciculi cruciati des Chiasma N. opt. bedingt. 
Auch hier wurde die Diagnose auf Hirntumor gestellt, wie in unseren beiden Fällen und dem Fall von Anuske. Im Gegensatz hierzu verlief der jetzt noch im Auszug zu beschreibende 4. Fall, von Eich horst ${ }^{1}$ ) mitgetheilt, zuerst unter dem Bilde einer acuten eitrigen idiopathischen cerebrospinalen Meningitis, deren Symptome unter allmählicher Entfieberung nachliessen; dann vielfache Exacerbationen und Remissionen. Nach drei Monaten Tod unter Steigerung der meningitischen Erscheinungen.

Anamnese: Stud. phil., 23 Jahre alt, bisher stets gesund; in den letzten Wochen wegen bevorstehenden Examens eifrigste Studien, denen er die Schuld an seiner jetzigen Erkrankung beimisst. Dieselbe begann am 5. Mai 1890 mit heftigem Schüttelfrost; darauf Hitzegefühl, starker Kopfschmerz; wiederholtes Erbrechen; der am nächsten Tage zugezogene Arzt constatirte bereits Nackensteifigkeit; in den nächsten Tagen Zunahme der Beschwerden; Bewusstsein noch benommen.

9. Mai 1890: Anfnahme in die Züricher medicinische Klinik.

Status. 9. Mai 1890. Sehr kräftig gebauter Mann. - Kopf tief nach hinten in die Kissen gebohrt. Somnolenz. Nur bei lautem Anreden langsame Antwort nach längerem Besinnen, die aber vernünftig und zutreffend ist; stöhnt und seufzt viel; quälender Kopfschmerz in Stirn-und Hinterhauptgegend. Haut heiss, trocken; Temperatur 40,1, Puls dabei 72, hie und da leicht unregelmässig. Athmung fast rein costal, sonst nicht abnorm. Pupillen eng, beiderseits gleich, träge reagirend. Bulbi nach allen Richtungen frei beweglich. Zunge wird unter Kraftanstrengung zögernd gerade herausgestreckt; Bew eg ungen des K opfes n ach vorn unmöglich; bei dem Versuch heftige Schmerzäusserung; Druck auf die Nackengegend sehr schmerzhaft; ophthalmoskopisch nichts Abnormes; Abdomen eingesunken; Druck auf die Beine überall sehr schmerzhaft; Patellarreflex normal; an den inneren Organen nichts $\mathrm{Ab}$ normes; Harn frei von Eiweiss und Zucker; Appetitlosigkeit, lebhafter Durst; Verstopfung. Diagnose: Meningitis cerebrospinalis purulenta idiopathica. Therapie: Eisblase auf Kopf und Nacken, Einlauf, Bromkali, Isolirung in verdunkeltem Zimmer, flüssige Kost.

Pat. hat Nachts delirirt. Ausgesprochene Lähmung des linken $\mathrm{Abducens}$ (Strab. conv. des linken Auges). Fieber zurückgehend, Harndrang, aber Strangurie. Nach 4 Tagen bedeutend geringeres Fieber. Kopfschmerz und Nackenstarre haben abgenommen, Blasenbeschwerden zugenommen (Katheterismus). Nach 2 Tagen bedentende Besserung, keine Harnbeschwerden, keine Delirien oder Somnolenz. - Am 19. Mai wieder stärkere Kopfschmerzen und Zunahme des Fiebers bis 39,0, das in den letzten 6 Tagen 38,0 nicht überschritten hatte; Phenacetin mildert die Kopfschmerzen. Am 25. Mai vermehrte Diurese, Zunahme der Kopfschmerzen. Im Harn nichts Abnormes. Am 30. Mai Kopfschmerzen verschwunden, Stuhlverstopfung. Fieber bedeutend geringer, remittirend. Am 2. Juni

1) Ueber den erworbenen jdiopathischen Hydrocephalus internus der Erwachsenen. Zeitschrift für klinische Medicin. Bd. XIX. Supplement. 
Nachts Delirien und zunehmender Kopfschmerz, ebenso am 5. Juni, dabei wieder hochgradige Nackenstarre; Leib stark eingezogen; Puls klein, unregelmässig; geringes remittirendes Fieber (38,0 Abends). Am 11. Juni Uebelkeit, Erbrechen. Seit 12. Juni Pat. benommen, unruhig, lässt mehrmals Harn unter sich. Ophthalmoskopie ergiebt ausser einer lebhaften geschlängelten Netzhautvene nichts Abnormes. Kein Fieber. Die nächsten 14 Tage hie und da leichte Temperatursteigerungen; Sensorium klarer; Kopfschmerzen geringer; Kopf freier beweglich. Am 28. Juni wieder Delirien, grosse Unruhe, stärkerer Kopfschmerz, häufiges Erbrechen. Am 30. Juni rechte Pupille dentlich weiter als linke. 6. Juli: Seit 6 Tagen kein Fieber, Kopfschmerzen geschwunden; Pupillen wieder gleich weit. Nackensteifigkeit unverändert. Subjectives Befinden gut. Am 19. Juli: Subjectives Befinden gut. Kopfschmerz nach tiefem Schlaf; Abends hie und da subfebrile Temperaturen. Am 28. Juli einmal Erbrechen. Bis zum 14. August subjectives Befinden sehr gut, selten kurzdauernde Kopfschmerzen und Erbrechen; Nackensteifigkeit unverändert. Am 15. August Morgens plötzlich ti efe Be nommenheit; Athmung unregelmässig, kein Cheyne-Stokes. Puls sehr verl ang s a mt, regelmässig; Nackenstarre verschwunden. Patellarreflex normal. - Nachmittags Exitus ohne besondere Erscheinungen.

Section. Schädel gross, von mittlerer Dicke; viel Diploë. Dura zart und trocken, Pia zart, durchscheinend; Hirnwindungen abgeflacht. Arachnoidea um das Infundibulum herum gelbweisslich verdickt; Infundibulnm selbst stark vorgetrieben, entleert beim Anstechen eine grosse Menge klar gelber Flüssigkeit (circa $300 \mathrm{Ccm}$.). Das ganze Hirn sehr voluminös, sinkt nach der Flüssigkeitsentleerung stark zusammen. Sämmtliche Gehirnhöhlen sehr stark erweitert. Foramen Monroi für den Mittelfinger durchgängig. Ependym im Ganzen glatt, nicht aufällig verdickt, zeigt zahlreiche, bis $2 \mathrm{Mm}$. breite, weisse, plättchenartige Verdickungen. Das ganze Gehirn schlaff und blass; Rinde nicht besonders verschmälert. Keine Localherde, - Die übrige Section ergiebt nichts Abnormes. Anatomische Diagnose: Hydroc. chron. int. congenitus.

Eichhorst knüpft an diesen Fall folgende epikritische Betrachtungen: "Wohl für Jeden wird das Ergebniss der Section, verglichen mit den klinischen Erscheinungen, ein ungewöhnlich auffälliges sein. Während des Lebens zweifelte man kaum einen Augenblick daran, dass man es mit einer ausgebildeten Meningitis zu thun habe; über ihre Natur dagegen konnte man schon verschiedener Meinung sein. Anfangs glaubten wir eine eitrige Meningitis vor uns zu haben, und es schien uns dafür der plötzliche Beginn und der stürmische Verlauf zu sprechen. Als sich dann aber die Körpertemperatur minderte und der Gang der Krankheit schleppender wurde, als sich mehrfach Remissionen und Exacerbationen bemerkbar machten, da wurde man begreiflicher Weise mehr und mehr unsicher, und immer stärker drängte sich die Annahme auf, dass wohl doch tuberculöse 
Veränderungen an den Meningen beständen. Der Mangel an Chorioideal-Tuberkeln, das Fehlen von tuberculösen Veränderungen in anderen Organen war nicht geeignet, die eben angedeutete Vermuthung: ausschliessen zu lassen. Die Section ergab weder das Eine noch das Andere, und merkwürdig genug, nicht einmal Zeichen einer vorausgegangenen Meningitis liessen sich mit Sicherheit erkennen. $\left.{ }^{1}\right)$ Niemand wird es daher dem Secirenden, welchem der klinische Verlauf unbekannt war, verargen, dass er seine anatomische Diagnose auf angeborenen inneren Hydrocephalus stellte. - Nach unserem Dafürhalten erklärt sich das Krankheitsbild am leichtesten, wenn man sich zu der Annahme bequemt, dass es einen selbständigen inneren Hydrocephalus der Erwachsenen giebt, welcher mitten in bester Gesundheit und sogor febril einsetzend das Bild einer Meningitis $\left.{ }^{2}\right)$ so genau wiedergeben kann, dass eine Unterscheidung während des Lebens unmöglich wird."

Dieser letzte Fall hat insofern einige Aehnlichkeit mit dem unseren ersten, als dort wie hier eine etwas auffallende Grösse des Schädels bestand, weshalb wir doch, zum Theil im Gegensatze zu Eichborst, zum Theil ïbereinstimmend mit dem jenen Fall Secirenden, als nicht ganz unwahrscheinlich annehmen möchten, dass ein gewisser Grad von Hydrocephalus internus congenital bestand, der dann aus uns unbekannten Ursachen erst im späteren Leben plötzlich so hochgradig wurde.

Beim Vergleiche dieser 5 Fälle wird eine grosse Aehnlichkeit in den klinischen Symptomen und dem Verlaufe unserer beiden, sowie der von Anuske und Oppenheim publieirten Fälle sofort auffallen, die sich aus dem bei dem betreffenden Sectionen iberall gefundenen, stark vergrösserten und nach unten sackförmig vorgetriebenen 3. Ventrikel erklärt, dureh welchen die seitlich und vorn von demselben verlaufenden Hirnnervenstämme mehr oder weniger gedrückt wurden. ${ }^{3}$ ) Alle Fälle verursachten die typischen Hirntumor-

1) Sollte nicht die ,um das Infundibulum herum gelbweisslich verdickte Arachnoidea" doch den Beweis einer vorher überstandenen Meningitis bilden?

2) oder eines Tumors.

3) Den anatomischen Verhältnissen nach sollte man die stärksten Ausfallserscheinungen von Seiten der Tract. opt., des Chiasma und der NN. oculomot. erwarten, wie dies hier auch zum grossen Theil der Fall. Warum meist der $\mathrm{N}$. abduc. mitbetheiligt, dagegen der $\mathrm{N}$. trochl. unbetheiligt bleibt, scheint nur darin begründet, dass der erstere durch die Vergrösserung des 3. Ventrikels gegen die innere Kante des Ganglion Gasseri gedrückt wird, während der letztere, begünstigt durch seine Dünne zwischen Oculomot. und Abduc., nach unten in den Sulcus caroticus auszuweichen vermag, dort vor Druck geschützt durch den freien Rand des Gezeltes, dicht unter welchem er verläuft (vgl. Hyrti, Lehrbuch der Anatomie des Menschen. Wien 1884. S.916). 
symptome, resp. alle die klinischen Symptome des allmählich zanehmenden Hirndrucks (Kopfschmerzen, Schwindel, Erbrechen, Pulsverlangsamung, Anfälle von Bewusstlosigkeit, zeitweise allgemeinere oder speciellere motorische Reizerscheinungen und Stauungspapille). Nur der Fall Eichhorst setzte unter dem Bild einer acuten cerebrospinalen Meningitis ein, um nachher bis zum Tod unter dem Bilde einer ehronischen Basalmeningitis zu verlaufen. Dieser ist auch der einzige Fall, bei dem jede Andeutung einer Staungspapille fehlt und nar ein Gehirnnerv Ausfallssymptome zeigte (der Abducens). Allen Fällen ist gemeinsam die mehr oder weniger ausgesprochene Nackenstarre, die nur in unserem 2. Fall fehlte, und vor Allem die öfteren plötzlich eintretenden Schwankungen in der Intensität des Hirndrucks, dessen plötzliche Zunahme sich äusserte in Obscurationen, Erweiterungen und Starre der Pupillen, Anfällen von Bewusstlosigkeit, theilweise mit voriubergehenden Reizerscheinungen wohl plötzlich vorübergehend gedrückter Hirnnervenstämme (NN. Facial. abduc. trochlear.) oder mehr allgemein motorischen (epileptiformen) Reizerscheinungen, ebenso wie dessen plötzliche Abnahme in dem Schwächerwerden oder Verschwinden dieser Symptome (alles aber Erscheinungen, die bei Hirntumoren ebenfalls häufig, seltener bei Meningitis beobachtet werden) ibren Ausdruck fand.

Die Erfahrungen aus den 5 angeführten Fällen zusammengefasst, könnte man somit doch nur zu dem Schlusse kommen, dass man beim Vorkommen eines ähnlichen Symptomencomplexes, wie in diesen Fällen, auch an die Möglichkeit eines idiopathischen Hydrocephalus internus der Erwachsenen denken müsse (nur die starken Hämorrhagien in der Retina sind bei Hirntumoren äusserst selten), wenn man auch niemals mit Sicherheit diese Diagnose wird stellen können, da ja Tumoren innerhalb der Schädelhöhle genau die gleichen Symptome machen können, sei es bei Mittelhirntumoren durch directen Druck oder directes Uebergreifen auf die Nachbarschaft, sei es beispielsweise bei Kleinhirntumoren durch den sehr stark und rasch sich entwickelnden begleitenden Hydrops, besonders des 3. Ventrikels. Dass auch eine Meningitis hierbei vorgetäuscht werden kann, beweist der Fall Eichhorst's.

Was unseren 2. Punkt, nämlich die seltene Complication eines Hydrocephalus internus oder auch nur einesraum. beschränkenden Processes in cerebro überhaupt') mit

1) Denn die Complication von idiopathischen Hydrocephalen der Erwachsenen mit Syringomyelie ist, wie bereits erwähnt, bisher überhaupt noch nicht beobachtet worden. 
Hydro-oder Syringomyelie anbetrifft, so existiren auch hierzu in der Literatur nur wenige Fälle, und zwar zum Theil solche, bei welchen entweder der Hydrocephalus intra vitam keine Symptome gemacht und erst bei der Autopsie als zufälliger Befund entweder für sich oder im Anschluss an eine Hirnerkrankung erwähnt wird, zum Theil solehe, wo die Syringomyelie ein zufälliger Befund war, oder endlich solche, wo wir es mit einer angeborenen gleichzeitigen Missbildung in Hirn und Rückenmark zu thun haben. In der diesbezïglichen Literatur*) konnte ich unter über 130 Fällen von Sy ringomyelie doch nur 15 dieser Art finden. (Es soll bei der nunmehr folgenden Aufzählung derselben stets zuerst die klinische Diagnose und dann der Seetionsbefund genannt werden.

1. Leydent): Meningocele. - P. m. Hydroe. int.; Hydromyelie.

2. Leyden ${ }^{1}$ ): Meningocele. - P. m. Hydroc. int.; Hydromyelie.

3. Schüle ${ }^{2}$ ): Progressive Paralyse. - P. m. Hydroc. int. (Gehirnrinde wenig atrophisch, Encephalitis, doppelseitige Opticusatrophie); Hydromyelie (Periependymitis spinalis. Myelitis der Hörner; diffuse Myelitis der Markstränge).

4. Eickhold ${ }^{3}$ ): Progressive Paralyse? - P. m. linsengrosses Lipom ûber dem linken Scheitellappen. Erweiterung der Seitenventrikel (grosse Ganglien und graue Hirnsubstanz, mässig atrophisch. Ependymitis). Hydromyelie (und Myelitis in deren Umgebung).

5. v. Lenhossek ${ }^{4}$ : Hydroe. chron. congenitus. - P. m. Hydroe. int.; Hydromyelie (nur in Med. obl. und Halsmark).

6. v. Lenhossek ${ }^{4}$ ): Malaria mit Hydrops universalis. - P. m. Ventrikelhydrops; Hydromyelie (nur in Med. obl. und Halsmark). (Centralkanal am Calamus scriptorius durch ein Con-

*) Am besten zusammengestellt in den sebr ausführlichen Arbeiten von:

A. Bäumler, Höhlenbildungen im Rückenmark. Deutsches Archiv für klinische Medicin. Bd. XL. S. $488 \mathrm{ff}$.

H. Chia ri, Ueber die Pathogenese der sogenannten Syringomyelie. Prager Zeitschr. f. Heilkunde. Bd. IX. S. $308 \mathrm{ff}$.

M. Miura, Zur Genese der Höhlen im Rückenmark. Virchow's Archiv f. pathologische Anatomie. Bd. 117. S. $446 \mathrm{ff}$.

M. Kiewlicz, Ein Fall von Myelitis transversa, Syringomyelie, multipler Sklerose und secundären Degenerationen. Deutsches Archiv für Psychiatrie. Bd. XX. S. $45 \mathrm{ff}$.

1) Virchow's Archiv. Bd. LXVIJI. S. 1. Beob. I. S. 11. Beob. II.

2) Deutsches Archiv f. klin. Med. Bd. XX. S. 271.

3) Archiv für Psychiatrie. Bd. X. S. 613.

4) Oesterr. Zeitschr. f. prakt. Heilkunde. Jahrg. V. Beob.I. S. 53. Beob. II. S. 58. 
glomerat von zerfallenen Ependymzellen verstopft. - Aortenstenose. Leber- und Milzvergrösserung.)

7. Langhan $\mathrm{s}^{5}$ ): Hirntumor. - P. m. Metastatisches Melanosarkom auf dem Boden des 4. Ventrikels. Ventrikel-Hydrops; Syringomyelie.

8. Langhans ${ }^{5}$ ): Hirntumor. - P. m. Sarkom des Wurms, hochgradiger Hydroc. int., Hydro- und Syringomyelie.

9. Langhan ${ }^{5}$ ): Hirntumor. - P. m. Sarkom des Plexus chorioideus quartus. Starker Hydroc. int.; Hydro- u. Syringomyelie.

10. Westphal ${ }^{6}$ ): Herderkrankung im Gehirn. - P. m. Tuberkel im Pons (am Boden des 4. Ventrikels links); Ventrikel-Hydrops; Hydro- und Syringomyelie.

11. Bamberger ${ }^{7}$ ): Progressive Muskelatrophie? - P. m. Hydrops der Ventrikel (Ependym verdickt); Hydromyelie.

12. Langhans ${ }^{8}$ ): Lepra anaesthetica. - P. m. Starker Hydrops der Ventrikel (Ependym verdickt); Syringomyelie (Erweichung der Hinterhörner, Clarke'schen Sänlen und grauen Commissur).

13. Kiewliecz ${ }^{9}$ ): Multiple Sklerose und Myelitis transversa. P. m. Multiple Hirn- und Riickenmarkssklerose (mit secundären Degenerationen), Syringomyelie. Starke Erweiterung der Seitènventrikel (frische Pachymeningitis. Myelitis transversa. - Leucocytose).

14. Chiari ${ }^{10)}$ : Hydroc. int. congenitus. - P. m. Hydroc. int. congenitus. Myelo-Meningocele lumbalis. Diastematomyelie und Syringomyelie.

15. Chiari ${ }^{10}$ ): Spina bifida. Hydroc. int. congenitus. - P. m. Starker Ventrikel-Hydrops. Starke Hydromyelie. Syringomyelie. Hydrencephalocele cerebello-cervicalis.

Ferner könnten noch erwähnt werden die 4 von Bäumler (l. c., S. 540) im Auszug mitgetheilten Fälle von congenitalem Hydroc. int. complicirt mit Spina bifida.

Bei Fall 1, 2, 5, 14 und 15 steht wohl Hydrocephalus und Hydromyelie insofern in einem ätiologischen Zusammenhang, als beide

5) Virch. Archiv, Bd. LXXXV. S.16. Beob. I. S. 18. Beob. II. S. 22. Beob. III.

6) Brain 1884. Vol. VI. S. 161. Beob. II.

7) Wiener med. Presse. Jahrg. X. 1869. Nr. 28. S. 650.

8) Virch. Archiv. Bd. 64. 1876. S. 175.

9) Archiv f. Psychiatrie. Bd. XX. 1889. S. 27.

10) Ueber Veränderungen des Kleinhirns infolge von Hydrocephalie des Grosshirns. Deutsche med. Wochenschr. 1891, Nr. 42. S. 1172 u. 1174. 
durch angeborene Bildungsfehler entstanden sind. Bei Fall 3, 4 und 11 handelt es sich wohl um einen Hydroc. int. ex vacuo, complicirt mit Hydromyelie aus unklarer Ursache. Bei Fall 6 könnte Hydrocephalus und Hydromyelie, wenn nicht beide eongenital, wohl eventuell ein weiteres Symptom des allgemeinen Hydrops sein (Eickholt erklärt beides aus serösem Erguss in Ventrikel und Centralkanal infolge von periependymärer Sklerose, bedingt durch die allgemeine Staung). Bei Fall 7, 8, 9 und 10 handelt es sich um Hirntumoren (mit begleitendem Ventrikelhydrops) und gleichzeitige Syringomyelie, die, trotzdem Langhans dieselbe als durch Anstaung der Cerebrospinalflüssigkeit von den im Gehirn statthabenden raumbeschränkenden Herden aus verursacht betrachtet, wohl als eine ganz selbständige Complication aufaufassen ist. Bei Fall 11 und 12 handelt es sich um einen Hydroc. int. aus unbekannter Ursache; im Fall 13 um VentrikelHydrops, wohl infolge der multiplen Sklerose im Gehirn; die gleichzeitig bestehende Syringomyelie dürfte von der Hirnveränderung völlig unabhängig sein. In unserem Falle ist die Aetiologie des Hydroc. int., oder vielleicht auch nur dessen plötzliches schnelles Zunehmen erst im höheren Alter, völlig unklar, dagegen kann wohl, wie wir zu erweisen versuchten, mit einiger Sicherheit angenommen werden, dass die gleichzeitig bestehende Syringomyelie eine congenitale Anomalie ist, wie auch das Bestehen eines gewissen Grades von angeborenem Hydroc. int. hier nicht unwahrscheinlich ist. Lehrt ja doch die Erfahrung, dass nicht gar selten Missbildungen im Gehirn mit solchen im Rüickenmark complicirt sind, wovon wir ja auch oben einige Beispiele angeführt haben. - Es findet sich jedoch unter den angeführten 15 Fällen kein einziger, bei dem es sich um die Complication eines idiopathischen Hydroc. int. der Erwachsenen mit Syringomyelie handelte; unser Fall ist somit in dieser Beziehung mit vollem Recht als ein Unicum zu bezeichnen.

So gering unsere Kenntniss in Betreff der Aetiologie des Hydroc. int. congenitus ist, ebensowenig wissen wir tiber die Ursache des Hydroc. int. der Erwachsenen. Huguenin ${ }^{1}$ ) sagt: „Es giebt im Kindesalter eine entzündliche Affection der Pia, welche in ihrer besonderen Artung noch sehr unklar ist; sie führt zu einem schneller oder langsamer entstehenden und wachsenden Ergusse in die Ventrikel, zur Ausweitung der letzteren; sie hat mit der Tuberculose nichts zu thun, von miliaren Granulationen findet sich keine

1) Ziemssen's Handbuch der speciellen Pathologie und Therapie Bd. XI. 1876. I. Hälfte. S. 420. 
Spur; ihre entzündliche Genese aber ist schwer zu demonstriren, dies ist der nicht tuberculöse Hydrocephalus acutus der Kinder. „Dieser Zustand kommt zur grössten Seltenheit auch bei Erwachsenen vor." (Huguenin bezeichnet den letzteren als ,einfache Basalmeningitis der Erwachsenen".) - Ebenso fasst auch Wernicke (l. c.), wie wir oben bereits erwähnt, diese seltenen Formen als Producte einer chronischen Leptomeningitis auf, bleibt uns aber ebenfalls den Beweis dafür schuldig. - Die Theorie, dass durch Traumen (z. B. schwere Gehirnerschütterungen; auch unser Patient hatte als Kind ein solches Trauma erlitten) in seltenen Fällen eine chronische Meningitis hervorgerufen werden könne, die dann zu dem Hydroc. Anlass giebt dadurch, dass diese meningitischen Processe sich hauptsächlich an der Basis und an den Plexus localisiren, ist ebensowenig bewiesen, wie diejenige, dass der Alkohol oder das syphilitische Virus an sich eine solche Meningitis hervorrufen könne. Da sich in diesen seltenen Fällen ausser einer leichten schwieligen Verdickung und Trübung der Dura, seltener der Pia, der Plexus und des Ependyms im Gehirn absolut nichts Pathologisches findet, was als Ursache des Hydrocephalus angesprochen werden könnte (weder sichere Zeichen einer abgelaufenen Meningitis, noch Venencompressionen oder Thrombosen, noch Verschluss des Foramen Monroi), und es in solchen Fällen , auch einer scharfsinnigen Spürlzunst nicht gelingt, ein ätiologisches Moment zu enthïllen"1), müssen wir eben zugestehen, vorläufig noch ausser Stande zu sein, diese merkwürdigen Vorgänge zu erklären. Ziegler ${ }^{2}$ ) meint, „dass die Affection (Entzündung der Meningen?) vielleicht manchmal mit einem Verschluss der in den queren Hirnspalten gelegenen Verbindungsöffnungen zwischen den Ventrikelhöhlen and den Subarachnoidalräumen zusammenhängt." - So ergiebt a u ch in unserem Falle selbst die eingehendste Untersuchung des Gehirns nicht das Geringste, was uns die plötaliche Entstehang, oder vielleicht auch nur die plötzliehe Zunahme des Hydrocephalus im Mannesalter erklären könnte; immerhin aber ist es nicht unwahrscheinlich, dass bei unserem Patienten ein geringer Grad von Hydroc. int. angeboren war, der erst im höheren Lebensalter aus uns allerdings unbekannter Ursache plötzlich zunahm (Patient hatte einen ziemlich grossen Schädel, der auch in seiner Kindheit schon aufgefallen war).

1) Heubner, Ueber Hydrocephalus. Eu lenburg's Realencyklopädie. 1887. Bd. IX. S. 667.

2) Lehrbu.ch der speciellen pathologischen Anatomie. Jena 1890. S. 320. 
Olme auf die Streitfrage näher einzugehen, welches eigentlich die Ursache der Spaltbildungen im Rückenmark sei, ob Gewebszerfall von entzündlichen Processen und Neubildungen, oder Erweiterungen des Centralkanals mit Ausstülpungen und secundären Abschntirangen (mit oder ohne Epithelverlust, angeboren oder erworben), möchte ich hier nun übersichtlich die Momente zusammenstellen, die seit der Kenntniss dieser Anomalien des Rückenmarks von den verschiedenen Autoren als ursächliche angegeben werden. ${ }^{1}$ ) Vorher sei noch erwähnt, dass $\mathrm{Chiari}^{2}$ ), der wohl am kritischsten die sämmtlichen Publicationen über Rückenmarkshöhlenbildungen beleuchtet und sichtet, nach eingehendster Betrachtung zu dem Schlusse kommt, ,dass in dem weitaus grösseren Theil der Fälle dieser Rückenmarkserkrankung die Höhlenbildung denn doch mit dem Centralkanale in irgend einer Weise im Zusammenhang stand", - und daher möchte, „dass man alle solchen Fälle sogenannter Syringomyelie durchweg als Hydromyelie bezeichnen sollte, gleichgültig, ob es sich um eine Dilatation des Centralkanals im Anschluss an eine Entwicklungsstörung oder Abspaltung: desselben, oder um eigentliche Dilatation des Centralkanals ohne diese Antecedentien handelt. Der Name Syringomyelie könnte dann noch immer in Verwendung bleiben für jene Fälle von langgestreckten Höhlenbildungen im Rückenmarke, welche sicherlich nicht mit dem Centralkanale in irgend einer Weise in Zusammenhang stehen. " 3 )

1) Aeusserst erschwert wird der Versuch, eine solche Uebersicht zu geben, einestheils durch die verschiedensinnige Anwendung der Worte: „Hydro- und Syringomyelie", anderentheils dadurch, dass es sich bei einer nicht ganz geringen Zahl von Spaltbildungen im Rückenmark um eine rorausgegangene embryonale Entwicklungsstörung handelte, die ihrerseits zu der Spaltbildung disponirte, welche dann erst auf irgend einen Reiz in der Nachbarschaft eintrat. Auch der Umstand, dass zwei Autoren (Simon und Wichmann, l. c.) angeben, dass in Zerfallsböhlen Epithelauskleidungen neu entstehen können, erschwert den Versuch einer solchen übersichtlichen Eintheilung, um nicht zu sagen, macht ihn fast unmöglich, so dass ich für die Fehler und Mängel in der nachfolgenden Zusammenstellung um Nachsicht bitten muss.

2) Zeitschrift f. Heilkunde. Prag 1888. Bd. IX. S. 324 u. 325.

3) Derselbe Autor lässt überhaupt nur 5 Formen der Spaltbildungen im Rückenmark gelten:

a) Erweiterung des Centralkanals nach vorausgegangener Entwicklungsstörung oder Abspaltung,

b) Erweiterung des Centralkanals ohne solche,

c) Höhlenbildung aus Destruction einer Gliawucherung,

d) Höhlenbildung mit myelitischer, resp. hämorrhagischer Genese,

e) Höhlenbildung durch regressiven Gewebszerfall. 
In das folgende Schema*) lassen sich alle Fälle nach den von den verschiedenen Autoren als ursächlich angegebenen Momenten geordnet einreihen.

1. Einfache Erweiterung des Centralkanals = Hydromyelie im engsten Sinne (Epithel völlig intact).

a) Angeboren 1) (Hemmungsbildung).

b) Erworben ${ }^{2}$ ) (allgemeine ${ }^{3}$ ) u. ${ }^{7}$ ) oder locale Störungen ${ }^{4}$ ), locale Compression $^{5}$ ), allgemeine Rückenmarksatrophie $\left.{ }^{6}\right)=$ Hydromyelus ex vacuo).

2. Erweiterung des Centralkanals mit Ausstülpungen und Divertikelbildungen, mit oder ohne secundäre Abschnïrung derselben $=\mathrm{Hy}-$ dromyelie im Sinne Leyden's (Epithel also völlig oder doch theilweise erhalten).

a) Angeboren ${ }^{7}$ ) (Hemmungsbildungen); hierber gehören wohl auch die Fälle von Verdoppelung oder Vervielfältigung des Centralkanals. ${ }^{8}$ )

b) Erworben (circumscripte oder diffuse, periependymäre, myelitische Sklerose ${ }^{9}$ ) mit durch die Gefässcompression vermehrter Transsudation oder centraler Erweicbung ${ }^{10}$ ), Erweichung des hypertrophirten Ependyms "), Stauung durch Tumoren in der hinteren Schädelgrube ${ }^{12}$ ), Narbenschrumpfung. ${ }^{13}$ )

3. Spaltbildung im Rückenmark (ohne Epithelauskleidung) $=$ Syringomyelie im engsten Sinn.

*) Die beigefügten Zahlen bezeichnen die der betreffenden Rubrik entsprechenden hier angeführten Fälle:

1) Hallopeau, Gaz. méd. de Paris. 1870. S. 183. - Sc hüle, 1. c. S. 271. M a der, Bericht der Kronprinz Rudolph-Stiftung pro 1884. S. 340.

2) Simon, Archiv für Psychiatrie. 1874. Bd. V. Beob. II. - Bamberger, 1. c. Nr. 28.

3) v. Lenhossek, 1. c. S. 58.

4) Langhans, Virchow's Archiv. 85. Bd. 1881. S. 1.

5) Eulenburg's Realencyklopädie. 1889. Bd. XVH. S. 62. - Wicham, Lancet 1881. Vol. I. p. 418.

6) Eickholt, 1. c. S. 613 .

7) Leyden, Klinik der Rückenmarkskrankheiten. 1875. Bd. II. S. 446. Derselbe, Virchow's Archiv. 1876. 68. Bd. S. 1 .

8) Kahler u. Pick, Prager Vierteljahrsschrift. 1879. Bd. CXXXXII. S. 20.

9) Hallop e au, l. c. S. $394,421,444,460$.

10) Joffroy et A chard, Arch. de phys. norm. et path. 1887.

11) Leyden, Virchow's Archiv. 68. Bd.

12) Langhans, l. c.

13) Grimm, Virchow's Archiv. 1869. 48. Bd. S. 445. 
[Simon ${ }^{14}$ ) and Wichmann ${ }^{15}$ ) äussern sogar die Ansicht, dass in Zerfallshöhlen im Rückenmark Epithelauskleidungen neu entstehen könnten, unabhängig vom Centralkanal (oder der letztere wenigstens erst secundär in Mitleidenschaft gezogen), der, meist vor der Höhle verlaufend, entweder obliterirt oder normal oder erweitert erhalten ist.]

a) Angeboren. ${ }^{16}$ )

b) Erworben

a) durch Gliomatose mit centralem Zerfall ${ }^{17}$ ),

$\beta)$ durch myelitische Processe mit centralem Zerfall ${ }^{18}$ ),

r) durch hämorrhagische Processe (mit Zertrümmerungen) oder embolische Processe (mit Erweichungen ${ }^{19}$ ), vgl. auch Leyden ${ }^{7}$ ),

d) durch regressiven Gewebszerfall. ${ }^{20}$ )

In unserem Falle haben wir es mit einer Spaltbildung zu thun, deren Ursache, wie wir gesehen haben, eine congenitale Anomalie ist, und möchte ich die gleichzeitig, aber nur in dem verlängerten Mark bestehende Erweiterung des Centralkanals nur als Effect der Einwirkung des sich fortpflanzenden starken Drucks der strotzend gefüllten Hirnventrikel auf den obersten Theil des Centralkanals betrachten (wie solche bei starkem Hydroc. int. öfter beobachtet ist), und nicht als eine Begleiterscheinung der Syringomyelie, wobei solche als ebenfalls vorkommend beschrieben ist. Eine Communication zwischen dem Centralkanal und der Spaltbildung konnte nirgends aufgefunden werden, ebensowenig wie irgend welche Reste von Epithel an den Wänden der Hôhlen; auch war der Centralkanal überall an normaler Stelle in einzelnen Schichten allerdings obliterirt isolirt za erkennen, so dass wir unseren Fall als eine echte Syringomyelie selbst im Sinne Chiari's bezeichnen können. ')

14) Simon, Archiv für Psychiatrie. 1874. Bd. V.

15) Wichmann, Geschwulst- und Höhlenbildung im Rückenmark. Monographie 1887.

16) Onser Fall $I$.

17) Simon, l. c. Beob. V, VII u. VIII. - Westphal, Archiv f. Psychiatrie. Bd. V. S. 98-99.

18) Vulpian, Arch. de phys. 1869. II Tome. p. 279. - Joffroy et Achard, l. c. Beob. II. - Sil c o ck, Rep. of path. soc. of London. Brit.med.journ. 1888. p.21.

19) Rullier, Journal de phys. exp. 1823. Obs. 121. - Monart, Arch. gén. de Méd. 1838. - Stadelmann, Deutsches Archiv für klinische Medicin. 1883. Ed. XXXIII. S. 126.

20) S teudener, Referat in Virchow-Hirsch's Jahresbericht. 1867. S. 404. Hitzig, Wiener med. Blätter. 1885. Nr. 42.

1) Vgl. Bäumler, 1. c., S. 469. - Kiewlicz, l. c., S. 21. 
Was die Aetiologie der Syringomyelien anbetrifft, so werden in der Literatur als häufigste Ursachen die Wirbelsäule treffende Traumen angeführt; auch in unserem Fall finden wir ein solches Trauma in der Anamnese verzeichnet, das aber die congenitale Höhle in ihrer Erweiterung höchstens unterstiitzt hat. Aber auch andere in der Literatur ${ }^{1}$ ) verzeichnete ätiologische Momente, wie Excesse in Baccho, Ueberanstrengung, Erkältung, Infectionskrankheiten ${ }^{2}$ ), finden wir in unserer Anamnese.

Der Umstand, dass in unserem Falle die Syringomyelie völlig symptomlos verlief, nimmt uns nicht mehr Wunder bei dem Ergebniss unserer mikroskopischen Untersuchung; sowie beim Durchmustern der diesbezüglichen Literatur, wo wir weit bedeutendere Höhlenbildungen (sowobl der Länge, als auch der Weite nach) im Rückenmark erwähnt finden, ohne dass während des Lebens die geringsten Symptome davon bestanden hätten. ${ }^{3}$ )

Hierher gehören die folgenden 9 Fälle, die ich des Interesses halber kurz zusammenstelle:

1. Arbeiter, bis zum 46. Jahre stets gesund; zu dieser Zeit schwere Verbrennung und Tod an Tetanus. - P. m. Ziemlich ausgedehnte (besonders in die Weite) Höhlenbildung im Rückenmark, im Dorsalmark auf Vorder- und Hinterhörner übergreifend.

2. Zimmermann, 50 Jahre alt, nie Rüekenmarkssymptome. Tod an Hirntuberkel. - P. m. Spaltbildung in der grauen Substanz des Rückenmarks und in den Hintersträngen.

3. Dienstmädehen, 21 Jahre alt, völlig gesund, stirbt plötzlich an Va-

1) Am übersichtlichsten zusammengestellt in der ausfübrlichen Dissertation von A. Bãumler, l. c., S. 480 .

2) Von für die Aetiologie der Syringomyelie in Betracht kommenden Infectionskrankheiten finden wir in der Literatur nur den acuten Gelenkrheumatismus erwähnt; in unserem Falle werden wir jedoch ausser auf diesen auch noch auf Diphtherie, Dysenterie, Cholera und Influenza anamnestisch hingewiesen.

3) Auch dies dürfte mit grösster Wahrscheinlichkeit dafür sprechen, dass in solchen Fällen die Spaltbildung c ongenital bestand, und dass durch sie oder ihre Vergrösserung während des Lebens (z. B. durch Traumen?) das umliegende Gewebe höchstens etwas zur Seite gedrängt, aber nicht oder nicht wesentlich in seiner Ernăhrung beeinträchtigt wird.

1. L. Clarke u. Radcliffe, On the path. of tetanus. Med. chir. transactions. 1865. Beob. II.

2. C. Westphal, l. c.

3., 4., 5. Th. Simon, Archiv für Psychiatrie. 1875. Bd. V. S. 132. Beob. V. S. 148. Beob. VI. S. 150. Beob. VII.

6, Kahler u. Pick, Beiträge zur Pathologie and pathologischen Anatomie des Centralnervensystems. Prag 1879. S. 105. Beob. I.

7., 8. A. Bäumler, 1. c., S. 444. Beob. I. S. 448. Beob. II.

9. Kiewlicz, l. c., S. 22 . 
riola. - P. m. Im ganzen Hals- und oberen Brustmark 2 kreisrunde Höhlungen von $6 \mathrm{Cm}$. Durchmesser.

4. Alte Frau ohne spinale Erkrankungssymptome. - P. m. Mit Cylinderepithel ansgekleidete von der Mitte des Brust- bis zum Ende des Lendenmarks hinabreichende Höble, die Rückenmarkssubstanz theilweise verdrängend.

5. 21 jähriger Commis, stets völlig gesund; Tod an Bronchopneumonie. - P. m. Höhlenbildungen vom verlängerten Mark bis zum Lendenmark reichend in der vorderen Partie der Hinterstränge; Degenerationen in den Hintersträngen; theilweise Angiome in den Hintersträngen.

6. Kräftiger Mann, von dem nervöse Störungen nicht bekannt. Tod an Zungenkrebs. - P. m. Syringomyelie.

7. 22 jähriges Dienstmädchen, stets gesund, Tod an Gesichtsrose. P. m. ziemlich ausgedehnte Syringomyelie, besonders im oberen Dorsalmark.

8. 29 jährige Maurersfrau ohne nervöse Beschwerden. Tod an progressiver, perniciöser Anämie. - P. m. Syringomyelie bedeutenden Grades, besonders in Höhe des 1 . bis 4 . und des 6 . bis 8 . Brustnervenabganges.

9. 22 jähriger Baner, stets gesund; Sturz von einem Baum; 3 Monate später erkrankt mit Rückenmarkssymptomen. Tod an grossem Decubitus und dessen Complicationen. - P. m. Syringomyelie, Myelitis transversa, multiple Gehirn- und Rückenmarks-Sklerose mit secundären Degenerationen.

So bot uns die Veröffentlichung unseres so ungemein seltenen Falles die Gelegenheit, auf verschiedene hochinteressante Abnormitäten aufmerksam zu machen: in erster Linie auf die Entwicklung eines idiopathischen Hydrocephalus internus beim Erwachsenen meis t unter typischen Hirntumorsymptomen - ein Fall, zu dem in der Literatur nur drei Parallelfälle sich auffinden liessen, und dem wir noch einen vierten, ebenfalls an der Freiburger Klinik beobachteten und noch nicht publicirten beifügen zu können in der Lage waren -, ferner auf die in der Literatur geradezu einzig dastehende Complication eines solchen Hydrocephalus mit Syringomyelie, die ibrerseits wieder in der Erklärung ihrer Entstehung (gegründet auf das Resultat einer eingehenden mikroskopischen Untersuchung) ganz vereinsamt in der Literatur über Spaltbildungen im Rückenmarke dasteht, und deren völlig symptomloser Verlauf ebenfalls zu den seltensten Vorkommnissen mit Recht zu zählen ist, da sich auch hiezu nur die neun erwähnten Parallelfälle in der gesammten in- und ausländischen Literatur finden liessen.

Zum Schlusse dieser Arbeit sei es mir gestattet, meinem hochverehrten früheren Chef, Herrn Geheimrath Prof. Dr. Bäumler, für die guitige Ueberlassung dieses Falles und die auf denselben bezüglichen werthvollen Winke, sowie Herrn Prof. v. Kahlden für die bereitwillige Unterstïtzung und Controle der pathologisch-anatomischen Untersuchungen meinen verbindlichsten Dank abzustatten. 


\section{Nachtrag.}

Leider war vorstehende Publication bereits druckfertig abge. schlossen, als in dieser Zeitschrift die Arbeit Hof f m a n n's ${ }^{1}$ ) erschien, welcher Autor nach zahlreich mitgetheilten Beobachtungen in Bezug auf die Aetiologie der Syringomyelie zu den gleichen Resultaten wie wir hier gekommen ist; nur spricht er die Ansicht aus, dass die Syringomyelie stets intra vitam diagnosticirbar sei und nur wegen anderer mehr hervorstechender accessorischer $\mathrm{K}$ rankheiten leicht übersehen werde, eine Thatsache, der unser in extenso publicirter Fall beweisend entgegentritt. ${ }^{2}$ ) Doch möge hier auf dieșe umfassende Arbeit, auf welche wir gern des Näheren eingegangen wären, nochmals hingewiesen werden.

1) Deutsche Zeitschrift für Nervenheilkunde. Bd. III. Heft I-III. 1892/93.

2) Es möge der Vollständigkeit halber noch kurz erwähnt werden, dass H off mann die Höhlenbildungen im Rückenmark folgendermaassen eintheilt:

I. Hydromyelus (latent oder mit unbekannten Symptomen verlaufend).

II. Primäre (centrale) Gliose mit und ohne Hydromyelie:

c) ohne Höhlenbildung (periependymäre Sklerose, periependymäre Myelitis, centrale Myelitis),

$\beta$ ) mit Spalt- und Höhlenbildung (Myelite cavitaire).

III. Centrale Gliomatose, mit oder ohne Höhlenbildung. 